\title{
Analog Least Mean Square Loop With I/Q Imbalance for Self-Interference Cancellation in Full-Duplex Radios
}

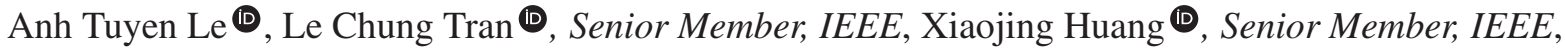 \\ and Yingjie Jay Guo $\mathbb{D}^{\mathbb{D}}$, Fellow, IEEE
}

\begin{abstract}
Analog least mean square (ALMS) loop is a promising structure for self-interference (SI) mitigation in full-duplex radios due to its simplicity and adaptive capability. However, being constructed from in-phase/quadrature (I/Q) demodulators and modulators to process complex signals, the ALMS loop may face I/Q imbalance problems. Thus, in this paper, the effects of frequencyindependent $\mathrm{I} / \mathrm{Q}$ imbalance in the ALMS loop are investigated. It is revealed that $I / Q$ imbalance affects the loop gain and the level of SI cancellation. The loop gain can be easily compensated by adjusting the gain at other stages of the ALMS loop. Meanwhile, the degradation on cancellation performance is proved to be insignificant even under severe conditions of $I / Q$ imbalance. In addition, an upper bound of the degradation factor is derived to provide an essential reference for the system design. Simulations are conducted to confirm the theoretical analyses.
\end{abstract}

Index Terms-Full-duplex, self-interference cancellation, ALMS loop, I/Q imbalance, analog imperfection.

\section{INTRODUCTION}

$\mathbf{R}$ ECENTLY, full-duplex (FD) transmission has gained a great attention due to its numerous advantages. By transmitting and receiving at the same time and in the same frequency band, this scheme can potentially double the throughput [1]. It also helps to bypass many intrinsic limitations in conventional cognitive radio networks due to simultaneous sensing and transmission [2]. Furthermore, this technique can be applied to other applications, such as synthetic aperture radar systems to overcome their fundamental limitations [3]. However, implementing FD transceivers faces a critical challenge of the strong self-interference (SI) caused by the co-located transmitter, which blocks the receiver from the desired signal propagated from the

Manuscript received February 6, 2019; revised May 10, 2019 and June 26 , 2019; accepted July 31, 2019. Date of current version October 18, 2019. This work was supported by the Australian Research Council's Discovery Project Funding Scheme under Project DP160101693. The review of this paper was coordinated by Dr. A. J. Al-Dweik. (Corresponding author: Anh Tuyen Le.)

A. T. Le, X. Huang, and Y. J. Guo are with the Faculty of Engineering and IT, University of Technology Sydney, Ultimo, NSW 2007, Australia (e-mail: anhtuyen.le@student.uts.edu.au; xiaojing.huang@uts.edu.au; jay.guo@uts. edu.au).

L. C. Tran is with the Faculty of Engineering and Information Sciences, University of Wollongong, Wollongong, NSW 2522, Australia (e-mail: lctran@uow.edu.au). far-end. Therefore, SI cancellation (SIC) is an essential issue in FD radios.

Self-Interference can be mitigated by passive suppression in the propagation domain and active cancellation in the analog and digital domains [4]. Passive methods aim to reduce the level of SI by isolating the transmitter and the receiver using antenna separation and different polarizations [5], [6]. Analog domain cancellation intends to create a signal, which mimics the SI, to cancel the SI after the receive antenna [7], [8]. Finally, in digital domain cancellation, the SI channel is estimated and combined with the transmitted baseband signal to cancel the residual SI after digitalized by the Analog-to-Digital converter (ADC) [9]. Although a combination of these cancellation approaches is always required to sufficiently remove the SI [10], analog domain cancellation has been proved to be the most important step of SI mitigation [11].

The reference signal for the analog SI cancellation can be mainly generated by two different methods. With the first method, the analog residual SI after cancellation is digitalized and processed by a complex algorithm to estimate the SI channel. The obtained information is then utilized to modify the baseband transmitted signal before it is up-converted and amplified by an additional transmit chain [12]-[14]. With the aid of digital signal processing (DSP), the level of SI can be reduced significantly in theory. However, capturing the residual SI in the digital domain as in [12]-[14] may lead to some distortions in practice. This is because an Automatic Gain Control amplifier, which is normally utilized to assure a wide dynamic range of a conventional receiver, will stabilize the level of the residual SI, resulting in an inaccurate reference signal synthesized by the DSP.

With the second method, the reference signal is generated directly at the radio frequency (RF) front-end by adjusting the amplitude and phase of the transmitted RF signal using a multi-tap structure. It has been proved that such a multi-tap adaptive structure is one of the most effective methods for wide-band applications [15]. However, unlike the cancellation performed in the digital domain where an adaptive filter can be simply implemented by a least mean square (LMS) algorithm, it is a significant challenge to realize the LMS loop in the RF domain since ideal RF integrators are hard to implement. To avoid this difficulty, the residual SI can be down-converted and 
digitalized by an additional receiver chain as in [15]-[18] to generate weighting coefficients for the LMS loop using the baseband integrators. The problem is that these additional blocks cause more interference and noise to the receiver. Furthermore, synthesizing weighting coefficients in the baseband stage is also impacted by hardware imperfections, such as I/Q imbalance in the transmission path [19]. As a result, a dedicated DSP is required to compensate for the hardware impairments.

A novel analog LMS (ALMS) loop proposed in [20] is a promising structure to avoid these problems. The ideal RF integrators can be replaced by simple resistor-capacitor lowpass filters (LPF) so that the weighting coefficients for the LMS algorithm are synthesized without sophisticated DSP. The studies on the behaviors of the ALMS loop published in [11], [20]-[22] are all conducted under the perfect I/Q imbalance-free condition of the loop. However, since I/Q imbalances always exist in the modulators and demodulators used in the ALMS loop, it is important to examine how these I/Q imbalances impact on the loop performance, which is the main motivation of this paper. To the best of the authors' knowledge, little work has been published in the literature considering this problem. Instead, most of the publications investigate the impact of I/Q imbalances appearing in the transceivers [9], [19], [23]-[25].

In this paper, the ALMS loop proposed in [20] with frequency independent I/Q imbalances will be investigated in both single carrier and orthogonal frequency division multiplexing (OFDM) FD systems. Firstly, the effect of I/Q imbalance on the loop gain is examined and compensated by adjusting the gains at other points in the loop. Secondly, the effect of I/Q imbalance on the cancellation performance is derived and quantified. Due to I/Q imbalance, the level of SIC given by the ALMS loop is degraded and can be presented by a degradation factor (DF). However, by averaging over random realizations of the SI channel, we show that the DF is insignificant even under severe amplitude and phase errors. In addition, an upper bound of the DF is also derived so that the maximum possible level of degradation can be analytically determined for a given I/Q imbalance condition. Hence, the contributions of this paper are threefold. First, it provides an analytical mean to investigate the impacts of the I/Q imbalance on the performance of the ALMS loop. Second, it proves the robustness of the ALMS loop as an effective SIC architecture against practical imperfections. Finally, it derives a closed-form DF upper bound which is critical from the system design perspective. This upper bound provides a reference for evaluating the SIC performance in the RF domain and also determining how much compensation should be gained from other SIC stages such as propagation domain suppression and digital domain cancellation to satisfy the overall SIC requirement.

The rest of this paper is organized as follows. In Section II, the system architecture and the signal models are described. Then, the effects of I/Q imbalance on the loop gain and cancellation performance are investigated in Section III. In Section IV, theoretical findings are verified by simulation results. Finally, conclusions are drawn in Section V.

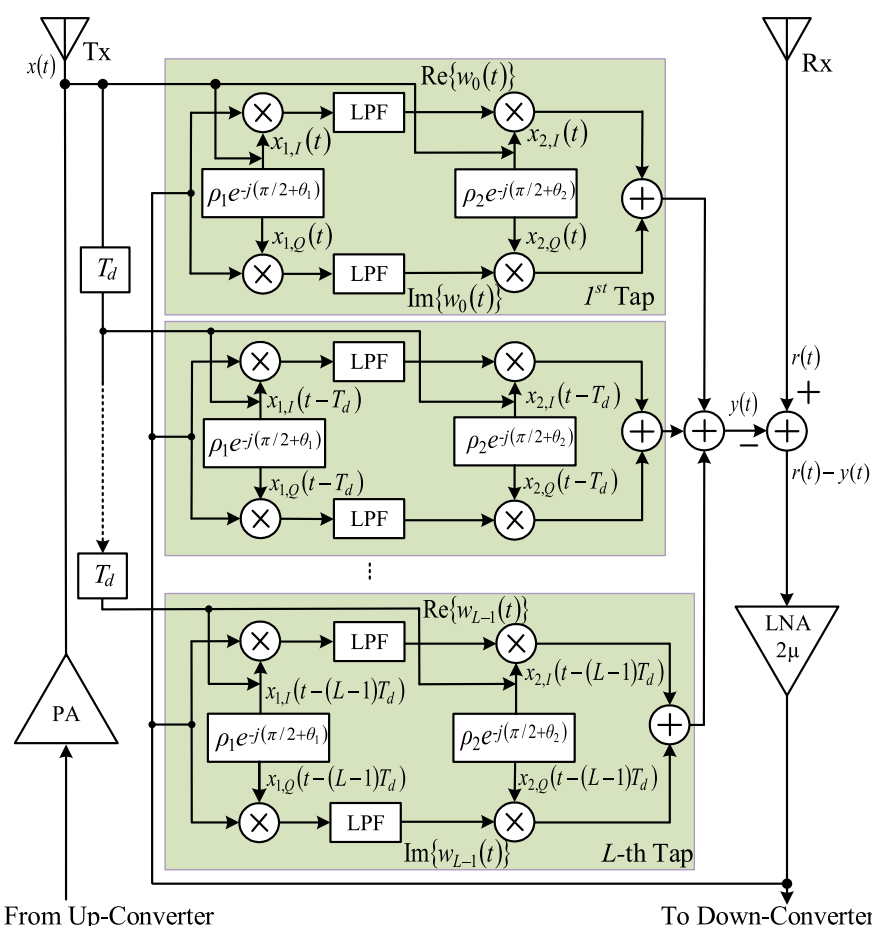

Fig. 1. The ALMS loop structure.

\section{System ARChitecture ANd Signal Models}

\section{A. Full-Duplex Transceiver With ALMS Loop}

Consider an FD transceiver terminal whose RF part is illustrated in Fig. 1. Owing to the FD operation, the received signal $r(t)$ is contaminated by a strong SI signal emitted from the local transmitter. In order to mitigate this SI, an ALMS loop is employed to generate a cancellation signal $y(t)$ and subtract it from the received signal at the input of the receiver. The ALMS loop, which comprises $L$ taps, processes complex signals using I/Q structures. At the $l$-th tap, the looped-back signal is multiplied with the delayed transmitted signal using an I/Q demodulator. LPFs at the output of the demodulator act as integrators to synthesize the weighting coefficients $w_{l}(t), l=0,1, \ldots, L-1$. These weighting coefficients then modulate the same delayed transmitted signal at the modulator. The cancellation signal $y(t)$ is generated by combining the outputs of all the taps. After subtracting the reference signal from the received signal $r(t)$, the residual signal is amplified by the LNA and looped-back to the input of every tap.

Signal models are expressed as follows. The RF transmitted signal $x(t)$ is expressed as $x(t)=\operatorname{Re}\left\{X(t) e^{j 2 \pi f_{c} t}\right\}$, where $f_{c}$ is the carrier frequency; $X(t)$ is the low-pass equivalent. Assume that the baseband part of this FD transceiver can operate in either single-carrier or multi-carrier such as OFDM modes. Therefore, $X(t)$ is denoted as $X(t)=X_{s}(t)$ and $X(t)=X_{o}(t)$ in the single-carrier and OFDM modes, respectively. $X_{s}(t)$ and $X_{o}(t)$ are modeled as

$$
X_{s}(t)=\sum_{i=-\infty}^{\infty} a_{i} q\left(t-i T_{s}\right)
$$


and

$$
\begin{aligned}
X_{o}(t)= & \sum_{n=-\infty}^{\infty} \sum_{m=-\infty}^{\infty} \sum_{k=-N_{s t} / 2, k \neq 0}^{N_{s t} / 2} a_{k, m} e^{j 2 \pi \frac{k}{N}\left(n-m \frac{T_{o}}{T_{s}}\right)} \\
& \cdot w\left[n-m \frac{T_{o}}{T_{s}}\right] q\left(t-n T_{s}\right)
\end{aligned}
$$

respectively, where $a_{i}$, and $a_{k, m}$ are the $i$-th data symbol in the single carrier system and the data symbol on the $k$-th sub-carrier of the $m$-th OFDM symbol respectively; $T_{s}$ is the symbol period of the single carrier system and also the sample period of the OFDM system; $T_{o}$ is the OFDM symbol duration; $N_{s t}$ is the total number of data subcarriers; $N$ is the number of samples in one OFDM symbol excluding cyclic prefix; $w[n]$ is the discrete windowing function applied to an OFDM symbol; and $q(t)$ is the pulse shaping function. The complex data symbols $a_{i}$ and $a_{k, m}$ are assumed to be independent to each other in both singlecarrier and OFDM systems, i.e.,

$$
E\left\{a_{i}^{*} a_{i^{\prime}}\right\}= \begin{cases}1, & \text { for } i=i^{\prime} \\ 0, & \text { for } i \neq i^{\prime}\end{cases}
$$

and

$$
E\left\{a_{k, m}^{*} a_{k^{\prime}, m^{\prime}}\right\}= \begin{cases}1, & \text { for } k=k^{\prime}, m=m^{\prime} \\ 0, & \text { for } k \neq k^{\prime}, m \neq m^{\prime} .\end{cases}
$$

where $E\{$.$\} stands for ensemble expectation. The root mean$ square amplitude of the transmitted signal is defined as $V_{X}=$ $\sqrt{\frac{1}{T} \int_{0}^{T} E\left\{|X(t)|^{2}\right\} d t}$ where $T$ is the period of transmitted data symbol, i.e., $T_{s}$ or $T_{o}$.

At the input of the receiver, the received signal $r(t)$ is a combination of the SI $z(t)$, the signal of interest $s(t)$, and the additive white Gaussian noise (AWGN) $n(t)$, i.e.,

$$
\begin{aligned}
r(t) & =z(t)+s(t)+n(t) \\
& =\operatorname{Re}\left\{[Z(t)+S(t)+N(t)] e^{j 2 \pi f_{c} t}\right\}
\end{aligned}
$$

where $Z(t), S(t)$ and $N(t)$ are the low-pass equivalents of these signals respectively. For the ease of derivation, the SI channel is modeled as an $L$ tapped delay line filter with tap delay $T_{d}$ as in the ALMS loop, i.e.,

$$
Z(t)=\sum_{l=0}^{L-1} h_{l}^{*} X\left(t-l T_{d}\right)
$$

where $h_{l}^{*}, l=0,1, \ldots L-1$, are the SI channel coefficients.

\section{B. I/Q Imbalanced Signal Models}

It can be seen from the architecture that the ALMS loop processes the complex signals directly in the analog domain and hence the quadrature demodulators and modulators are required in the loop. Assume that the I/Q imbalances of the demodulators and modulators are frequency-independent, and hence the phase shifters in the demodulators and modulators have constant amplitudes $\rho_{1}$ and $\rho_{2}$ as well as phase errors $\theta_{1}$ and $\theta_{2}$, respectively. Considering the demodulator of the first tap, the complex transmitted signal, denoted as $x_{1}(t)$ and presented at the demodulator, can be modeled as $x_{1}(t)=x_{1, I}(t)+j x_{1, Q}(t)$ where $x_{1, I}(t)$ and $x_{1, Q}(t)$ are the real transmitted signals at the I-branch and Q-branch respectively, and expressed as

$$
\begin{aligned}
x_{1, I}(t) & =\operatorname{Re}\left\{X(t) e^{j 2 \pi f_{c} t}\right\} \\
& =\frac{1}{2}\left[X(t) e^{j 2 \pi f_{c} t}+X^{*}(t) e^{-j 2 \pi f_{c} t}\right] \\
x_{1, Q}(t) & =\operatorname{Re}\left\{\rho_{1} X(t) e^{j\left(2 \pi f_{c} t-\pi / 2-\theta_{1}\right)}\right\} \\
& =\operatorname{Im}\left\{\rho_{1} X(t) e^{j\left(2 \pi f_{c} t-\theta_{1}\right)}\right\} \\
& =\frac{1}{2 j}\left[\rho_{1} X(t) e^{j\left(2 \pi f_{c} t-\theta_{1}\right)}-\rho_{1} X^{*}(t) e^{-j\left(2 \pi f_{c} t-\theta_{1}\right)}\right] .
\end{aligned}
$$

Hence, the complex signal $x_{1}(t)$ is represented, in terms of the baseband equivalent $X(t)$, as

$$
\begin{aligned}
x_{1}(t) & =x_{1, I}(t)+j x_{1, Q}(t) \\
& =\frac{1+\rho_{1} e^{-j \theta_{1}}}{2} X(t) e^{j 2 \pi f_{c} t}+\frac{1-\rho_{1} e^{j \theta_{1}}}{2} X^{*}(t) e^{-j 2 \pi f_{c} t} .
\end{aligned}
$$

At the $l$-th tap, the product of $x_{1}\left(t-l T_{d}\right)$ and the looped-back signal is filtered by the LPF to attain the weighting coefficient, denoted as $w_{l}(t)$, which can be derived as [20], [26]

$$
\begin{aligned}
w_{l}\left(t+t_{0}\right)=\frac{2 \mu \alpha}{K_{1} K_{2}} \int_{t_{0}}^{t+t_{0}} & e^{-\alpha\left(t+t_{0}-\tau\right)} \\
& \times[r(\tau)-y(\tau)] x_{1}\left(\tau-l T_{d}\right) d \tau
\end{aligned}
$$

where $K_{1}$ and $K_{2}$ are the dimensional constants of the multipliers in the demodulator and modulator respectively, $2 \mu$ is the gain of the LNA, $\alpha=1 / R C$ is the decay constant of the resistor-capacitor LPF with resistance $R$ and capacitance $C$, and $0 \leq t_{0}<T$ is an initial starting time. Similarly, the complex signal $x_{2}(t)$, presented in the modulator, can be expressed as

$x_{2}(t)=\frac{1+\rho_{2} e^{-j \theta_{2}}}{2} X(t) e^{j 2 \pi f_{c} t}+\frac{1-\rho_{2} e^{j \theta_{2}}}{2} X^{*}(t) e^{-j 2 \pi f_{c} t}$ (7)

Hence, the cancellation signal $y(t)$ at the output of the ALMS loop can be expressed as

$$
y(t)=\operatorname{Re}\left\{\sum_{l=0}^{L-1} w_{l}^{*}(t) x_{2}\left(t-l T_{d}\right)\right\} .
$$

For simplicity, we denote $\eta_{i}=\frac{1+\rho_{i} e^{-j \theta_{i}}}{2}$ and $\xi_{i}=$ $\frac{1-\rho_{i} e^{j \theta_{i}}}{2}$, for $i=1$,2. From (6) and (7), $y(t)$ can be further 
expressed as

$$
\begin{aligned}
y(t)=\operatorname{Re} & \left\{\sum _ { l = 0 } ^ { L - 1 } w _ { l } ^ { * } ( t ) \left[\eta_{2} X\left(t-l T_{d}\right) e^{j 2 \pi f_{c}\left(t-l T_{d}\right)}\right.\right. \\
& \left.\left.+\xi_{2} X^{*}\left(t-l T_{d}\right) e^{-j 2 \pi f_{c}\left(t-l T_{d}\right)}\right]\right\} .
\end{aligned}
$$

Clearly, the cancellation signal is affected by the I/Q imbalances at both modulators and demodulators.

\section{EFFECTS OF I/Q IMBALANCE}

\section{A. Impact on Loop Gain}

It has been proved in [20]-[22] that the loop gain, which is jointly determined by the LNA gain, the transmitted signal amplitude and the dimensional constants of the multipliers in the modulator and demodulator, plays a vital role in the performance of the ALMS loop. The loop gain $G$ is defined as

$$
G=\frac{V_{y}}{V_{d}}
$$

where $V_{y}$ and $V_{d}$ are the RMS voltages of the cancellation signal at the output and the residual SI signal at the input of the ALMS loop (before the LNA) respectively. From the loop architecture, when no I/Q imbalance presents, the loop gain $G$ is calculated as

$$
G=\frac{V_{y}}{V_{d}}=\frac{\frac{2 \mu V_{d} V_{x}}{K_{1}} \frac{V_{x}}{K_{2}}}{V_{d}}=\frac{2 \mu V_{x}^{2}}{K_{1} K_{2}}
$$

where $V_{x}$ is the RMS voltage of the RF signal $x(t)$.

Since the I/Q imbalance causes the errors in phase and amplitude of the signals, it also impacts the total loop gain. The loop gain in the case of the I/Q imbalance, denoted as $G^{\prime}$, can be calculated as

$$
G^{\prime}=\frac{V_{y}}{V_{d}}=\frac{\frac{2 \mu V_{d} V_{x_{1}}}{K_{1}} \frac{V_{x_{2}}}{K_{2}}}{V_{d}}=\frac{2 \mu V_{x_{1}} V_{x_{2}}}{K_{1} K_{2}}
$$

where $V_{x_{1}}$ and $V_{x_{2}}$ are the RMS voltages of the I/Q imbalanced RF transmitted signals at the demodulator and modulator respectively. The signal powers $P_{x}$ and $P_{x_{i}}($ for $i=1,2)$ are derived as

$$
\begin{gathered}
P_{x}=V_{x}^{2}=\frac{1}{T} \int_{0}^{T} E\left\{x^{2}(t)\right\} d t=\frac{1}{2 T} \int_{0}^{T} E\left\{|X(t)|^{2}\right\} d t \\
P_{x_{i}}=V_{x_{i}}^{2}=\frac{1}{2 T} \int_{0}^{T} E\left\{\mid \eta_{i} X(t) e^{j 2 \pi f_{c} t}\right. \\
\left.\quad+\left.\xi_{i} X^{*}(t) e^{-j 2 \pi f_{c} t}\right|^{2}\right\} d t \\
=\frac{1}{2 T} \int_{0}^{T} E\left\{\left[\eta_{i} X(t) e^{j 2 \pi f_{c} t}+\xi_{i} X^{*}(t) e^{-j 2 \pi f_{c} t}\right]\right. \\
\left.\quad \cdot\left[\eta_{i}^{*} X^{*}(t) e^{-j 2 \pi f_{c} t}+\xi_{i}^{*} X(t) e^{j 2 \pi f_{c} t}\right]\right\} d t \\
=\frac{1}{2 T} \int_{0}^{T} E\left\{\left(\left|\eta_{i}\right|^{2}+\left|\xi_{i}\right|^{2}\right)|X(t)|^{2}\right. \\
\left.\quad+2 \operatorname{Re}\left\{\eta_{i} \xi_{i}^{*} X^{2}(t) e^{j 4 \pi f_{c} t}\right\}\right\} d t .
\end{gathered}
$$

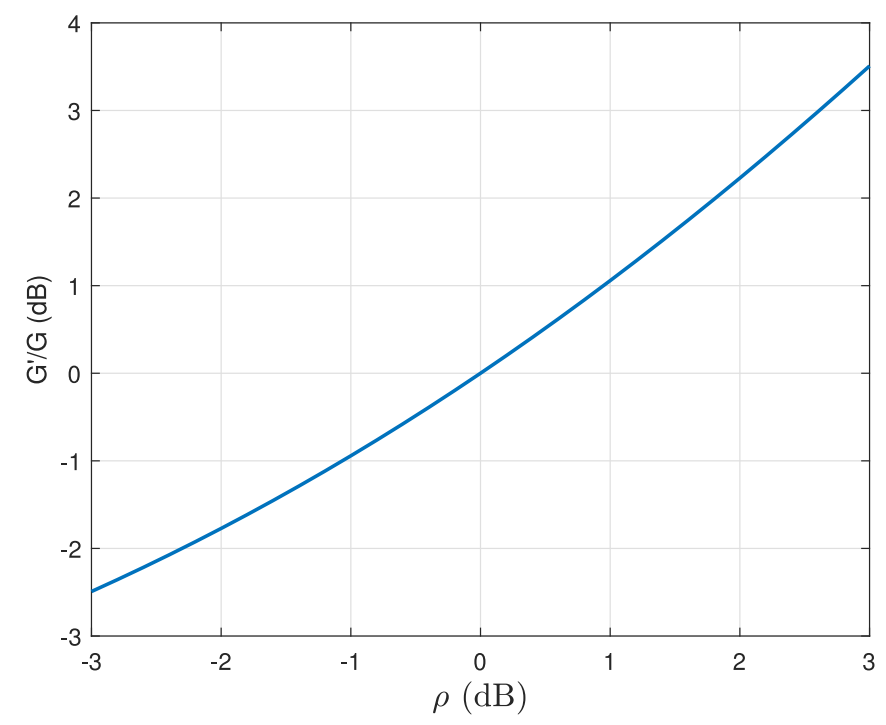

Fig. 2. The loop gain ratio versus amplitude.

Since $E\left\{X^{2}(t)\right\}=0$ for any zero mean complex random process $X(t)$, we have

$$
\begin{aligned}
P_{x_{i}} & =\frac{\left(\left|\eta_{i}\right|^{2}+\left|\xi_{i}\right|^{2}\right)}{2 T} \int_{0}^{T} E\left\{|X(t)|^{2}\right\} d t \\
& =\left(\left|\eta_{i}\right|^{2}+\left|\xi_{i}\right|^{2}\right) P_{x} .
\end{aligned}
$$

Therefore, the effect of I/Q imbalance on the loop gain can be represented by

$$
\begin{aligned}
G^{\prime} & =\sqrt{\left(\left|\eta_{1}\right|^{2}+\left|\xi_{1}\right|^{2}\right)\left(\left|\eta_{2}\right|^{2}+\left|\xi_{2}\right|^{2}\right)} G \\
& =\frac{1}{2} \sqrt{\left(1+\rho_{1}^{2}\right)\left(1+\rho_{2}^{2}\right)} G .
\end{aligned}
$$

We can see that the change on the loop gain is a function of the amplitudes of the phase shifters in the demodulators and the modulators only. Assume that the phase shifters in the demodulators and modulators have the same amplitudes, i.e., $\rho_{1}=\rho_{2}=\rho$, the ratio $G^{\prime} / G=\frac{1+\rho^{2}}{2}$ over a range of $\rho$ is presented in Fig. 2. From this figure, the amplitude errors may contribute to the loop gain $(\rho>0 \mathrm{~dB})$ or reduce the loop gain $(\rho<0 \mathrm{~dB})$. The variation of the loop gain can be compensated accordingly by changing the gain at other stages in the loop such as the LNA.

\section{B. Impact on Cancellation Performance}

In this section, we investigate the impact of I/Q imbalance on the cancellation performance of the ALMS loop. In order to analyze the impact of I/Q imbalance under the same loop gain, the gain of the LNA $2 \mu$ can be scaled to $2 \mu^{\prime}$ with $\mu^{\prime}=$ $\frac{2 \mu}{\sqrt{\left(1+\rho_{1}^{2}\right)\left(1+\rho_{2}^{2}\right)}}$. The following derivation will be conducted with this adjustment of the LNA gain. The residual SI power in case of the ALMS loop with I/Q imbalance is compared to that in the perfect case of no I/Q imbalance. In this analysis, the ALMS loop is considered in the steady state, i.e., all the weighting coefficients are converged to their stable values. Therefore, both ensemble expectation and time averaging, denoted as $\bar{E}\{$.$\} , are$ 


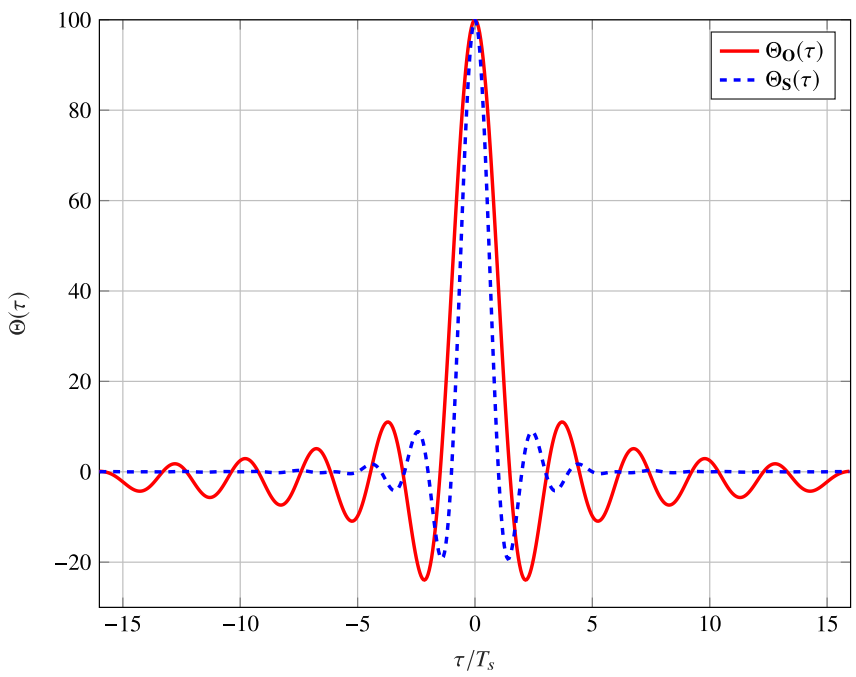

Fig. 3. The normalized autocorrelation function of the transmitted signals.

applied to evaluate all the processes. The normalized autocorrelation functions of the transmitted signal, denoted as $\Theta(\tau)$, is defined as

$$
\begin{aligned}
\Theta(\tau) & =\frac{1}{K_{1} K_{2}} \bar{E}\left\{X^{*}(t) X(t-\tau)\right\} \\
& =\frac{1}{K_{1} K_{2} T} \int_{0}^{T} E\left\{X^{*}(t) X(t-\tau)\right\} d t .
\end{aligned}
$$

Denoting $A^{2}=V_{X}^{2} / K_{1} K_{2}, \Theta(0)=\frac{1}{K_{1} K_{2} T} \int_{0}^{T} E\left\{|X(t)|^{2}\right\} d t$ $=A^{2}$, which is the normalized power of the transmitted signal. Fig. 3 demonstrates the normalized autocorrelation functions of the single carrier signal $\Theta_{\mathrm{S}}(\tau)$ and OFDM signal $\Theta_{\mathrm{O}}(\tau)$ with $A^{2}=100$. In this plot, $q(t)$ is a root-raised cosine (RRC) function in the single carrier case and raised cosine (RC) function for the OFDM case. The windowing function for the OFDM signal is the one recommended in IEEE 802.11a. All pulse shaping filters and windowing function have the roll-off factor $\beta=0.25$.

Defining the residual SI signal as $d(t)=z(t)-y(t)$, using the signal models in (3) and (8), and denoting $u_{l}(t)=h_{l}$ $\left(\eta_{2}^{*} w_{l}(t)+\xi_{2} w_{l}^{*}(t)\right) e^{j 2 \pi f_{c} l T_{d}}$, we can express $d(t)$ as

$$
\begin{aligned}
& d(t)=z(t)-y(t) \\
& =\operatorname{Re}\left\{\sum_{l=0}^{L-1} h_{l}^{*} X\left(t-l T_{d}\right) e^{j 2 \pi f_{c} t}\right\}-\operatorname{Re}\left\{\sum_{l=0}^{L-1} w_{l}^{*}(t)\right. \\
& \left.\cdot\left[\eta_{2} X\left(t-l T_{d}\right) e^{j 2 \pi f_{c}\left(t-l T_{d}\right)}+\xi_{2} X^{*}\left(t-l T_{d}\right) e^{-j 2 \pi f_{c}\left(t-l T_{d}\right)}\right]\right\} \\
& =\frac{1}{2}\left[\sum_{l=0}^{L-1} u_{l}^{*}(t) X\left(t-l T_{d}\right) e^{j 2 \pi f_{c} t}\right. \\
& \left.+\sum_{l=0}^{L-1} u_{l}(t) X^{*}\left(t-l T_{d}\right) e^{-j 2 \pi f_{c} t}\right] \\
& =\operatorname{Re}\left\{\sum_{l=0}^{L-1} u_{l}^{*}(t) X\left(t-l T_{d}\right) e^{j 2 \pi f_{c} t}\right\} .
\end{aligned}
$$

It means that the residual SI of the ALMS loop is determined by the weighting error function $u_{l}(t)$. The normalized residual SI power, denoted as $P_{d}(t)$, can be determined as

$$
P_{d}(t)=\frac{1}{K_{1} K_{2}} \bar{E}\left\{|d(t)|^{2}\right\} .
$$

Substituting (17) into (18), after some manipulations (see Appendix A) and let $t \rightarrow \infty, P_{d}(t)$ converges to its steady-state

$$
P_{d}=\frac{1}{2} \overline{\overline{\mathbf{u}}}^{H} \Theta \overline{\overline{\mathbf{u}}}
$$

where

$\Theta=\left[\begin{array}{cccc}\Theta(0) & \Theta\left(-T_{d}\right) & \cdots & \Theta\left(-(L-1) T_{d}\right) \\ \Theta\left(T_{d}\right) & \Theta(0) & \cdots & \Theta\left(-(L-2) T_{d}\right) \\ \vdots & \vdots & \ddots & \vdots \\ \Theta\left((L-1) T_{d}\right) & \Theta\left((L-2) T_{d}\right) & \cdots & \Theta(0)\end{array}\right]$

is the $L \times L$ autocorrelation matrix and $\overline{\overline{\mathbf{u}}}=\left[\overline{\bar{u}}_{0} \cdots \overline{\bar{u}}_{L-1}\right]^{T}$ is the vector of the steady-state weighting error functions. From the solution of the weighting error function (see Appendix B), $\overline{\overline{\mathbf{u}}}$ is expressed as

$$
\begin{aligned}
\overline{\overline{\mathbf{u}}}= & {\left[\left(\mathbf{I}_{L}+k_{1} \boldsymbol{\Theta}\right)-\left|k_{2}\right|^{2} \mathbf{E} \Theta\left(\mathbf{I}_{L}+k_{1}^{*} \boldsymbol{\Theta}\right)^{-1} \mathbf{E}^{-1} \boldsymbol{\Theta}\right]^{-1} } \\
& \cdot\left[\mathbf{h}-k_{2} \mathbf{E} \Theta\left(\mathbf{I}_{L}+k_{1}^{*} \boldsymbol{\Theta}\right)^{-1} \mathbf{h}^{*}\right]
\end{aligned}
$$

where $\mathbf{I}_{L}$ is the identity matrix of order $L ; k_{1}=\mu^{\prime}\left(\eta_{1} \eta_{2}^{*}+\right.$ $\left.\xi_{1}^{*} \xi_{2}\right)$, and $k_{2}=\mu^{\prime}\left(\eta_{2}^{*} \xi_{1}+\eta_{1}^{*} \xi_{2}\right)$ are constants representing the $\mathrm{I} / \mathrm{Q}$ imbalance errors;

$$
\mathbf{E}=\left[\begin{array}{cccc}
1 & 0 & \cdots & 0 \\
0 & e^{j 4 \pi f_{c} T_{d}} & \cdots & 0 \\
\vdots & \vdots & \ddots & \vdots \\
0 & \cdots & 0 & e^{j 4 \pi f_{c}(L-1) T_{d}}
\end{array}\right]
$$

and $\mathbf{h}=\left[h_{0} h_{1} \cdots h_{L-1}\right]^{T}$ is the channel coefficients of $L$ paths.

When there is no I/Q imbalance, i.e., $\rho_{1}=\rho_{2}=1, \theta_{1}=\theta_{2}=$ 0 , thus $k_{1}=\mu, k_{2}=0$, we have $\overline{\overline{\mathbf{u}}}=\left(\mathbf{I}_{L}+\mu \boldsymbol{\Theta}\right)^{-1} \mathbf{h}$. Note that the autocorrelation matrix can be decomposed as $\Theta=\mathbf{Q} \mathbf{\Lambda} \mathbf{Q}^{-1}$ where $\mathbf{Q}$ is the orthonormal modal matrix whose columns are the $L$ eigenvectors of $\Theta$ and

$$
\Lambda=\left(\begin{array}{cccc}
\lambda_{0} & 0 & \cdots & 0 \\
0 & \lambda_{1} & \cdots & 0 \\
\vdots & \vdots & \ddots & \vdots \\
0 & 0 & \cdots & \lambda_{L-1}
\end{array}\right)
$$

is a diagonal spectral matrix, and $\lambda_{l}, l=0, \ldots, L-1$, are $L$ eigenvalues of $\Theta$. In this case, the residual SI is calculated as

$$
\begin{aligned}
P_{0} & =\frac{1}{2} \mathbf{h}^{H}\left(\mathbf{I}_{L}+\mu \boldsymbol{\Theta}\right)^{-1} \boldsymbol{\Theta}\left(\mathbf{I}_{L}+\mu \boldsymbol{\Theta}\right)^{-1} \mathbf{h} \\
& =\frac{1}{2} \mathbf{h}^{H} \mathbf{Q}\left(\mathbf{I}_{L}+\mu \boldsymbol{\Lambda}\right)^{-1} \mathbf{Q}^{-1} \mathbf{Q} \mathbf{\Lambda} \mathbf{Q}^{-1}\left(\mathbf{I}_{L}+\mu \boldsymbol{\Lambda}\right)^{-1} \mathbf{Q}^{-1} \mathbf{h} \\
& =\frac{1}{2} \mathbf{h}^{H} \mathbf{Q} \operatorname{diag}\left\{\frac{\lambda_{l}}{\left(1+\mu \lambda_{l}\right)^{2}}\right\} \mathbf{Q}^{-1} \mathbf{h}
\end{aligned}
$$




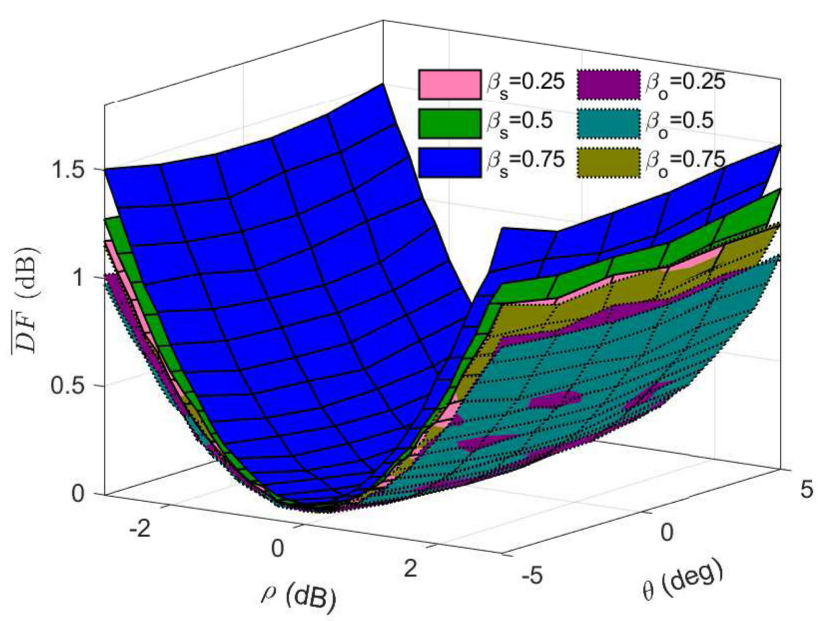

(a)

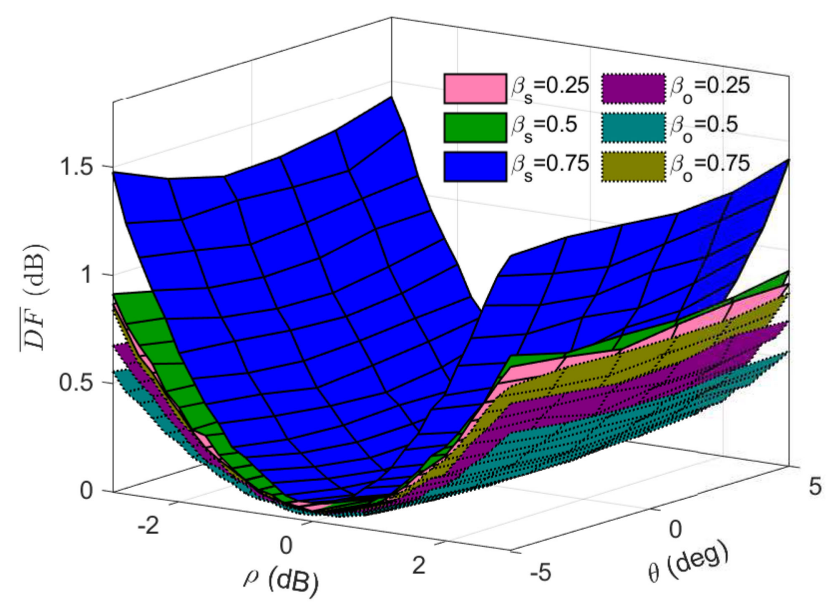

(b)

Fig. 4. $\overline{D F}$ versus amplitude and phase errors with different $\beta$ for $L=8$ and (a) $f_{c}=2432 \mathrm{MHz}$ and (b) $f_{c}=5200 \mathrm{MHz}$.

where $\operatorname{diag}\left\{\frac{\lambda_{l}}{\left(1+\mu \lambda_{l}\right)^{2}}\right\}, l=1, \ldots, L$, is an $L \times L$ diagonal matrix whose main diagonal elements are $\frac{\lambda_{1}}{\left(1+\mu \lambda_{1}\right)^{2}}, \ldots, \frac{\lambda_{L}}{\left(1+\mu \lambda_{L}\right)^{2}}$. We can see that $P_{0}$ is the same as the result derived in [20, Eq. (37)]. From (19) and (21), when there are I/Q imbalances present, we will see that I/Q imbalances of the modulators and demodulators not only lead to the change of the residual SI power, but also result in the involvement of the carrier frequency in the performance of the ALMS loop.

To quantify the impact of I/Q imbalance on the level of cancellation, we define the degradation factor $D F$ as

$$
\begin{aligned}
D F & =10 \log _{10} \frac{P_{d}}{P_{0}} \\
& =10 \log _{10} \frac{\overline{\overline{\mathbf{u}}}{ }^{H} \boldsymbol{\Theta} \overline{\overline{\mathbf{u}}}}{\mathbf{h}^{H} \mathbf{Q} \operatorname{diag}\left\{\frac{\lambda_{l}}{\left(1+\mu \lambda_{l}\right)^{2}}\right\} \mathbf{Q}^{-1} \mathbf{h}} .
\end{aligned}
$$

From (20) and (22), in addition to the I/Q imbalance, the $D F$ is also related to other factors such as the SI channel $\mathbf{h}$, the transmitted signal properties (shown in $\Theta$ ), and the carrier frequency of the transmitted signal (shown in the matrix $\mathbf{E}$ ). Therefore, two questions can be raised at this point. First, what is the $D F$ on average over many realizations of the SI channel? Second, what is the possible maximum level of the $D F$ ? The latter is very important as it provides an analytical upper bound useful for the system design. These two questions are addressed as follows.

1) Averaged Degradation Factor: To evaluate the overall impact of the SI channel in the $D F$, we further define an averaged degradation factor $\overline{D F}$ as

$$
\overline{D F}=10 \log _{10} \frac{E_{h}\left\{P_{d}\right\}}{E_{h}\left\{P_{0}\right\}}
$$

where $E_{h}\{$.$\} stands for expectation over the SI channel.$

Assume that proper propagation domain suppression has been performed so that the SI mainly comes from multipath reflections. All the coefficients of the SI channel which includes $L$ propagation paths are independent and Gaussian distributed with a zero mean. An exponential distribution is chosen for the power delay profile of the channel. Its root mean square delay spread is selected as $\sigma=L T_{s} / 4$. The transmitter has symbol duration $T_{s}=20 \mathrm{~ns}$. The ALMS loop has the tap delay $T_{d}=T_{s} / 2$. When operating in the multi-carrier mode, the parameters of the transmitted OFDM signal are selected following the IEE802.11a standard. For simplicity, we assume that the phase shifters in the modulators and demodulators have the same degree of imperfections, i.e., $\rho_{1}=\rho_{2}=\rho$ and $\theta_{1}=\theta_{2}=\theta$. Fig. 4 and Fig. 5 present $\overline{D F}$ obtained by averaging over 10000 realizations of the SI channel over a range of the amplitude $(-3 \mathrm{~dB} \leq \rho \leq 3 \mathrm{~dB})$ and phase error $\left(-5^{\circ} \leq \theta \leq 5^{\circ}\right)$ for the systems with carrier frequencies of $2432 \mathrm{MHz}$ and $5200 \mathrm{MHz}$, respectively. These two carrier frequencies are chosen as examples because they are common worldwide IEEE 802.11 frequency bands. In Fig. 4, $\beta_{\mathrm{s}}$ and $\beta_{\mathrm{o}}$ stand for the roll-off factors of the pulse shaping filter and the windowing function in the single carrier and OFDM basebands, respectively. In Fig. 5, all the roll-off factors are fixed at $\beta=0.25$ and $\overline{D F}$ is calculated for different number of taps $L$ in the ALMS loop.

From these figures, some observations are drawn as follows.

1) $\overline{D F}$ is more impacted by the amplitudes than the phase errors. We can see that $\overline{D F}$ is almost stable over the whole range of $\theta$ for a given value of $\rho$.

2) When $\theta=0$ and $\rho=1, \overline{D F}=0 \mathrm{~dB}$, i.e., there is no $\mathrm{I} / \mathrm{Q}$ imbalance.

3) Signal properties $\left(\beta\right.$ and $f_{c}$ ) have more influence on $\overline{D F}$ than the number of taps in the loop.

4) $\overline{D F}$ does not exceed $1.5 \mathrm{~dB}$ for most of the cases. It is slightly greater than $1.5 \mathrm{~dB}$ when $\beta_{\mathrm{s}} \geq 0.75$. However, these conditions unlikely happen in practice.

It can be concluded that, considering the average effect of the SI channel, the ALMS loop is resilient to its frequencyindependent I/Q imbalance. This resilience can be explained as follows. The ALMS loop is designed to minimize the level of the residual SI power. The adaptive adjustment of the weighting coefficients ensures that the ALMS loop always works at its optimized condition. Therefore, the impact of the I/Q imbalance 


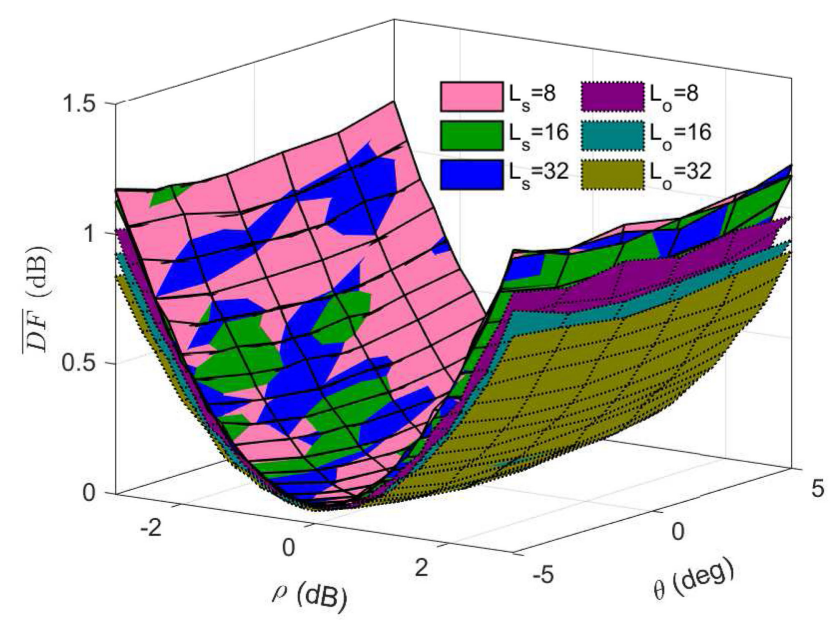

(a)

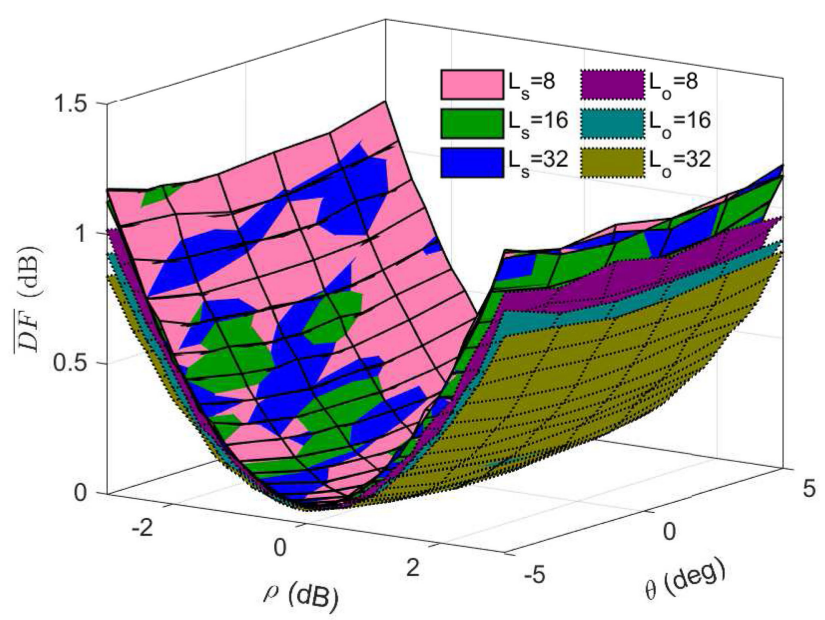

(b)

Fig. 5. $\overline{D F}$ versus amplitude and phase errors with different $L$ for $\beta=0.25$ and (a) $f_{c}=2432 \mathrm{MHz}$ and (b) $f_{c}=5200 \mathrm{MHz}$.

occurred in the ALMS loop on the SIC performance is not serious as that of the I/Q imbalance occurred in the signal transmission path on the information detection performance.

2) Upper Bound of Degradation Factor: From the system design perspective, it is essential to estimate the level of degradation of SIC given by the ALMS loop under a given condition of I/Q imbalance. This degradation of SIC in the RF domain can be compensated at other stages such as propagation domain attenuation or digital domain cancellation. Therefore, we introduce the upper bound of the degradation factor, denoted as $D F_{u}$, which can be derived as follows.

In the ideal case when the roll-off factor of the pulse shaping filter is zero, the tap delay $T_{d}$ can be chosen as $T_{d}=T_{s}$. In this case, the autocorrelation matrix $\Theta$ is a diagonal matrix and all of its eigenvalues are equal, i.e., $\lambda_{0}=\lambda_{1}=\cdots=\lambda_{L-1}=\bar{\lambda}=A^{2}$. Therefore, from (20), the weighting error functions $\overline{\overline{\mathbf{u}}}$ becomes

$$
\begin{aligned}
\overline{\overline{\mathbf{u}}}= & {\left[\left(\mathbf{I}_{L}+k_{1} \Theta\right)-\left|k_{2}\right|^{2} \mathbf{E} \Theta\left(\mathbf{I}_{L}+k_{1}^{*} \boldsymbol{\Theta}\right)^{-1} \mathbf{E}^{-1} \boldsymbol{\Theta}\right]^{-1} } \\
& \cdot\left[\mathbf{h}-k_{2} \mathbf{E} \Theta\left(\mathbf{I}_{L}+k_{1}^{*} \boldsymbol{\Theta}\right)^{-1} \mathbf{h}^{*}\right] \\
= & {\left[\left(1+k_{1} A^{2}\right)-\frac{\left|k_{2}\right|^{2} A^{4}}{1+k_{1}^{*} A^{2}}\right]^{-1}\left[\mathbf{h}-\frac{k_{2} A^{2}}{1+k_{1}^{*} A^{2}} \mathbf{E h}^{*}\right] } \\
= & \kappa\left[\left(1+k_{1}^{*} A^{2}\right) \mathbf{h}-k_{2} A^{2} \mathbf{E} \mathbf{h}^{*}\right]
\end{aligned}
$$

where $\kappa=\left[1+2 \operatorname{Re}\left\{k_{1}\right\} A^{2}+\left(\left|k_{1}\right|^{2}-\left|k_{2}\right|^{2}\right) A^{4}\right]^{-1}$. Substituting (24) into (19), we have

$$
\begin{aligned}
P_{d}= & \frac{1}{2} \kappa^{2} A^{2}\left[\left(1+k_{1} A^{2}\right) \mathbf{h}^{H}-k_{2}^{*} A^{2} \mathbf{h}^{T} \mathbf{E}^{-1}\right] \\
& \cdot\left[\left(1+k_{1}^{*} A^{2}\right) \mathbf{h}-k_{2} A^{2} \mathbf{E h}^{*}\right] \\
= & \frac{1}{2} \kappa^{2} A^{2}\left[\left(1+2 \operatorname{Re}\left\{k_{1}\right\} A^{2}+\left(\left|k_{1}\right|^{2}+\left|k_{2}\right|^{2}\right) A^{4}\right) \sum_{l=0}^{L-1}\left|h_{l}\right|^{2}\right.
\end{aligned}
$$

$$
\begin{aligned}
& -2\left\{| k _ { 2 } ^ { * } A ^ { 2 } ( 1 + k _ { 1 } ^ { * } A ^ { 2 } ) | \sum _ { l = 0 } ^ { L - 1 } | h _ { l } | ^ { 2 } \operatorname { c o s } \left(-4 \pi f_{c} l T_{d}\right.\right. \\
& \left.\left.\left.+2 \phi_{h_{l}}+\phi_{k}\right)\right\}\right]
\end{aligned}
$$

where $\phi_{h_{l}}$ and $\phi_{k}$ are the phases of the SI channel coefficient $h_{l}$ and the complex number $k_{2}^{*} A^{2}\left(1+k_{1}^{*} A^{2}\right)$, respectively. Since $-1 \leq \cos (x) \leq 1$ for any angle $x$ and $\operatorname{Re}\{z\} \leq|z|$ for any complex number $z$, we have the upper bound of the residual SI power as

$$
\begin{aligned}
P_{d} \leq \frac{1}{2} \kappa^{2} A^{2}[ & \left(1+2 \operatorname{Re}\left\{k_{1}\right\} A^{2}+\left(\left|k_{1}\right|^{2}+\left|k_{2}\right|^{2}\right) A^{4}\right) \sum_{l=0}^{L-1}\left|h_{l}\right|^{2} \\
& \left.+2\left\{\left|k_{2}^{*} A^{2}\left(1+k_{1}^{*} A^{2}\right)\right| \sum_{l=0}^{L-1}\left|h_{l}\right|^{2}\right\}\right] \\
\leq \frac{1}{2} \kappa^{2} A^{2}\left[1+2\left(\left|k_{1}\right|+\left|k_{2}\right|\right) A^{2}\right. & \\
& \left.+\left(\left|k_{1}\right|^{2}+2\left|k_{1} k_{2}\right|+\left|k_{2}\right|^{2}\right) A^{4}\right] \sum_{l=0}^{L-1}\left|h_{l}\right|^{2} \\
=\frac{1}{2} \kappa^{2} A^{2}[1 & \left.+\left(\left|k_{1}\right|+\left|k_{2}\right|\right) A^{2}\right]^{2} \sum_{l=0}^{L-1}\left|h_{l}\right|^{2} .
\end{aligned}
$$

Under the same condition, $P_{0}$ is found to be $P_{0}=$ $\frac{A^{2}}{2\left(1+\mu A^{2}\right)^{2}} \sum_{l=0}^{L-1}\left|h_{l}\right|^{2}$. Therefore, $D F_{u}$ can be found as

$$
\begin{aligned}
D F_{u} & =10 \log _{10} \frac{\frac{1}{2} \kappa^{2} A^{2}\left[1+\left(\left|k_{1}\right|+\left|k_{2}\right|\right) A^{2}\right]^{2} \sum_{l=0}^{L-1}\left|h_{l}\right|^{2}}{\frac{A^{2}}{2\left(1+\mu A^{2}\right)^{2}} \sum_{l=0}^{L-1}\left|h_{l}\right|^{2}} \\
& =10 \log _{10} \frac{\left[1+\left(\left|k_{1}\right|+\left|k_{2}\right|\right) A^{2}\right]^{2}\left(1+\mu A^{2}\right)^{2}}{\left[1+2 \operatorname{Re}\left\{k_{1}\right\} A^{2}+\left(\left|k_{1}\right|^{2}-\left|k_{2}\right|^{2}\right) A^{4}\right]^{2}} .
\end{aligned}
$$

Assuming that $\mu A^{2}$ is very large so that $1 / \mu A^{2} \approx 0$, and by dividing both numerator and denominator in (27) by $\left(\mu A^{2}\right)^{4}$, we 


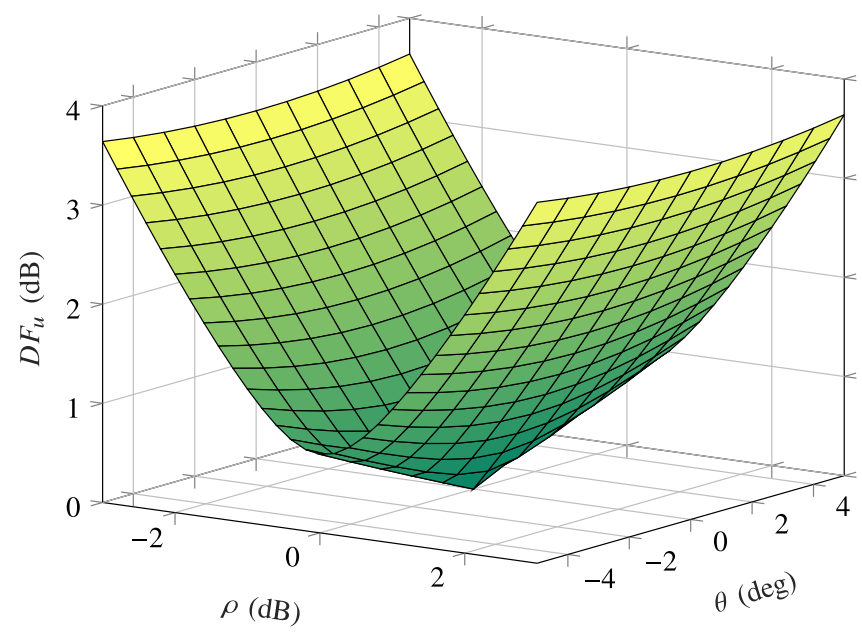

Fig. 6. $D F$ versus amplitude and phase errors when $\beta=0$ and $T_{d}=T_{s}$.

have

$$
D F_{u}=10 \log _{10} \frac{\frac{\left(\left|k_{1}\right|+\left|k_{2}\right|\right)^{2}}{\mu^{2}}}{\left(\frac{\left|k_{1}\right|^{2}-\left|k_{2}\right|^{2}}{\mu^{2}}\right)^{2}} .
$$

Let the phase shifters in the modulators and demodulators have the same degree of imperfections, i.e., $\rho_{1}=\rho_{2}=\rho$ and $\theta_{1}=\theta_{2}=\theta$. Substituting the definition of $k_{1}, k_{2}$ and $\mu^{\prime}=2 \mu /\left(1+\rho^{2}\right)$ into (28), we have

$$
\begin{aligned}
D F_{u} & =10 \log _{10} \frac{\frac{\mu^{2}\left(1+\rho^{2}+\sqrt{1-2 \rho^{2} \cos 2 \theta+\rho^{4}}\right)^{2}}{\left(1+\rho^{2}\right)^{2} \mu^{2}}}{\left(\frac{\mu^{2}\left[\left(1+\rho^{2}\right)^{2}-\left(1-2 \rho^{2} \cos 2 \theta+\rho^{4}\right)\right]}{\left(1+\rho^{2}\right)^{2} \mu^{2}}\right)^{2}} \\
& =20 \log _{10} \frac{\left(1+\rho^{2}\right)\left(1+\rho^{2}+\sqrt{1-2 \rho^{2} \cos 2 \theta+\rho^{4}}\right)}{4 \rho^{2} \cos ^{2} \theta} .
\end{aligned}
$$

Fig. 6 presents $D F_{u}$ calculated from (29) as a function of $\rho$ and $\theta$. We can see that $D F$ is mainly determined by the amplitudes, while it is almost the same over the range of phase errors. Hence, when selecting the modulators and demodulators for the ALMS loop, it is better to choose those with small amplitude errors.

In addition, in the worst scenarios when $\rho= \pm 3 \mathrm{~dB}, D F_{u}$ is about $3.5 \mathrm{~dB}$ only. This $3.5 \mathrm{~dB}$ deterioration of SIC can be compensated at other stages such as propagation domain suppression and digital domain cancellation.

\section{Simulation Results}

In this section, simulations are performed to justify the theoretical results shown in Section III. The FD system with configuration as in Fig. 1 is employed with QPSK modulation and $T_{s}=20 \mathrm{~ns}$. When operating in the multi-carrier mode, the OFDM symbol of duration $T_{o}=80 T_{s}$ is generated from 64-point IFFT with cyclic prefix added. The OFDM symbols are then passed through the IEEE 802.11a windowing function and the RC pulse shaping filter before sending to the RF frontend. All the roll-off factors of the pulse shaping filter and windowing

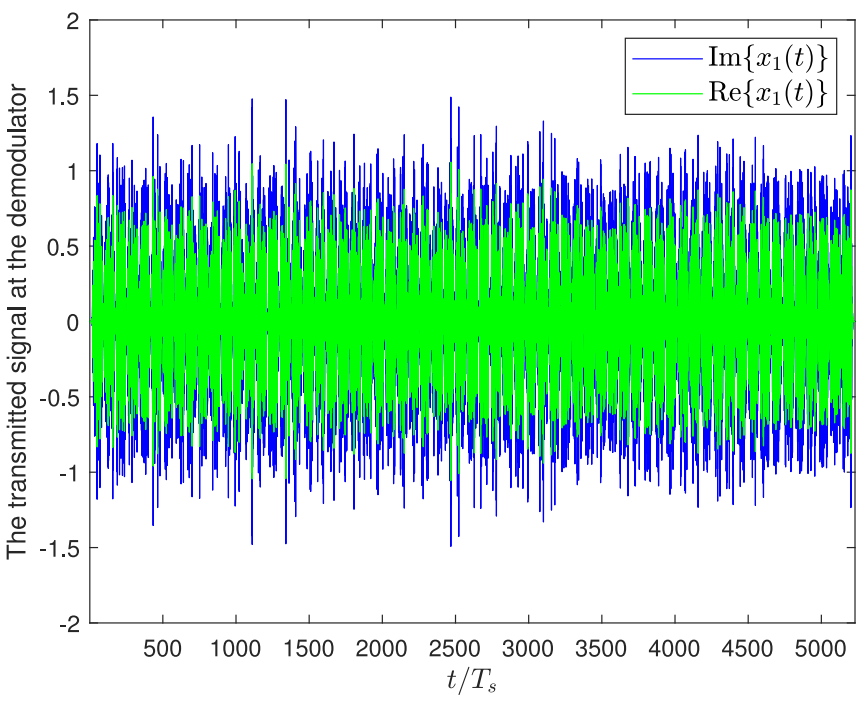

Fig. 7. The complex signals $x_{1}(t)$ and $x_{2}(t)$ with $\rho_{1}=\rho_{2}=3 \mathrm{~dB}$ $\theta_{1}=\theta_{2}=5^{\circ}$.

function are $\beta=0.25$. The RF transmitted signal has power of $0 \mathrm{dBm}$ over a $50 \mathrm{Ohm}$ load or $0 \mathrm{dBm}+10 \log _{10}(50)=17 \mathrm{dBm}$ over $1 \mathrm{Ohm}$ load. Hence, the RMS voltage of the (baseband) transmitted signal for $1 \mathrm{Ohm}$ load can be found as $V_{X}^{2}=$ $2 \times 10^{(17-30) / 10}=0.1 V^{2}$. The ALMS loop has 8 taps with the tap spacing $T_{d}=T_{s} / 2$. The demodulators and modulators in all taps have the same multiplier constants which are selected as $K_{1} K_{2}=0.001 \mathrm{~V}^{2}$. Due to the separation of the transmit and receive antennas, the SI power at the input of the receiver is attenuated $25 \mathrm{~dB}$ compared to the transmitted signal power. The channel impulse response presented in [20, Fig. 10] is adopted in this paper for simulation. Accordingly, the SI channel includes three paths and is modeled as $h(t)=10^{\frac{-25}{20}}\left\{\left[\frac{\sqrt{2}}{2}-\right.\right.$ $\left.0.5 j] \delta(t)-0.4 \delta\left(t-T_{s}\right)+0.3 \delta\left(t-3 T_{s}\right)\right\}$, i.e., the delays of reflected paths are multiples of $T_{s}$. The LNA in the receiver is designed so that its gain can be varied in order to compensate for the change of the total loop gain. In case of no I/Q imbalance, the LNA gain $2 \mu$ is selected as $26 \mathrm{~dB}$, i.e., $\mu=10$.

The modulators and demodulators in the ALMS loop are chosen such that their phase shifters have the same I/Q imbalance parameters as $\rho_{1}=\rho_{2}=\rho=3 \mathrm{~dB}, \theta_{1}=\theta_{2}=\theta=5^{\circ}$. Due to these imperfections, the OFDM transmitted signals presented at the modulators and demodulators are illustrated in Fig. 7. To compare the performance under the same loop gain, the LNA gain is adjusted to $\mu^{\prime}=2 \mu /\left(1+\rho^{2}\right)=6.6772$ when I/Q imbalance present at the ALMS loop.

Fig. 8 and Fig. 9 show the simulated results (blue curves) in comparison to the theoretical results for carrier frequencies of $f_{c}=2432 \mathrm{MHz}$ and $f_{c}=5200 \mathrm{MHz}$, respectively. The greendashed line is the theoretical residual SI power (cf. (19)) for the case of the ALMS loop with I/Q imbalance. The red line is the residual SI power (cf. (21)) for the case of the ALMS loop under an I/Q imbalance-free condition. It can be seen from these figures that the convergence of the simulation results (blue curves) to the theoretical results (green-dashed lines) confirms 


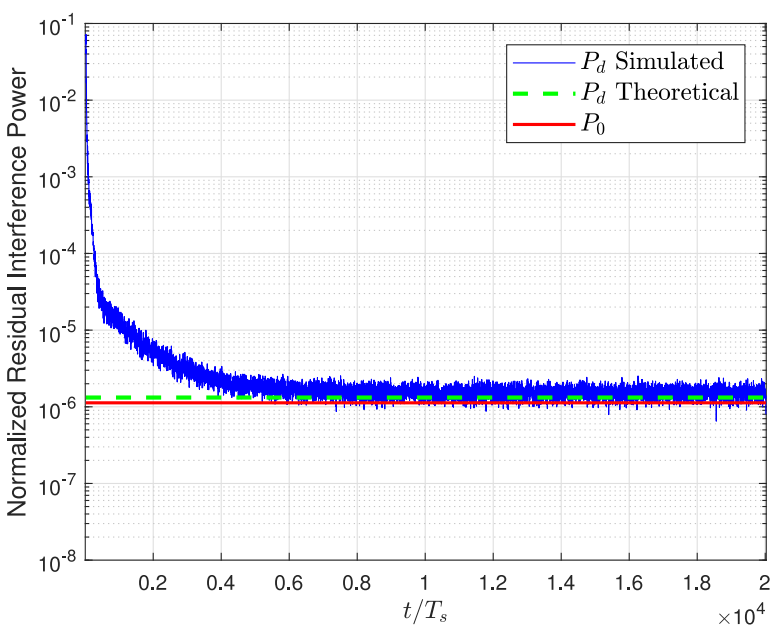

(a)

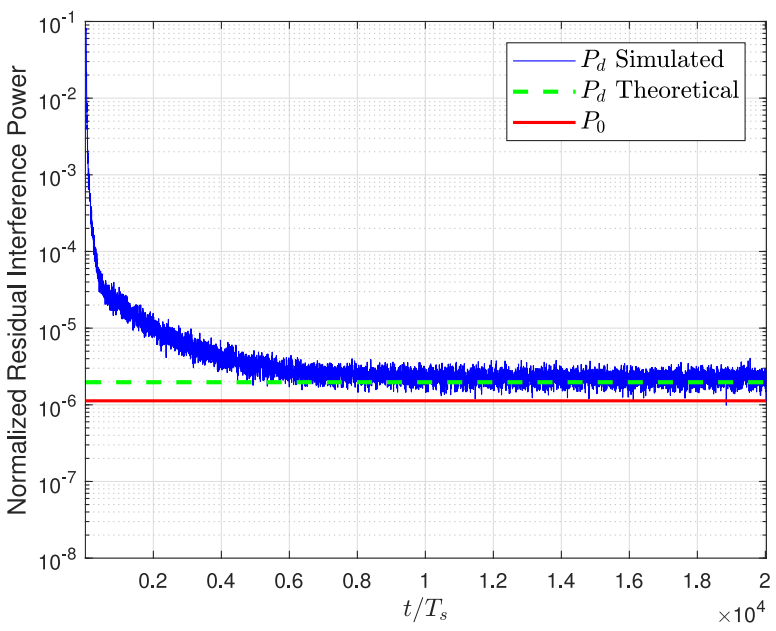

(b)

Fig. 8. Residual SI powers in single carrier system with $\rho_{1}=\rho_{2}=3 \mathrm{~dB}, \theta_{1}=\theta_{2}=5^{\circ}$ and (a) $f_{c}=2432 \mathrm{MHz}$ and (b) and $f_{c}=5200 \mathrm{MHz}$.

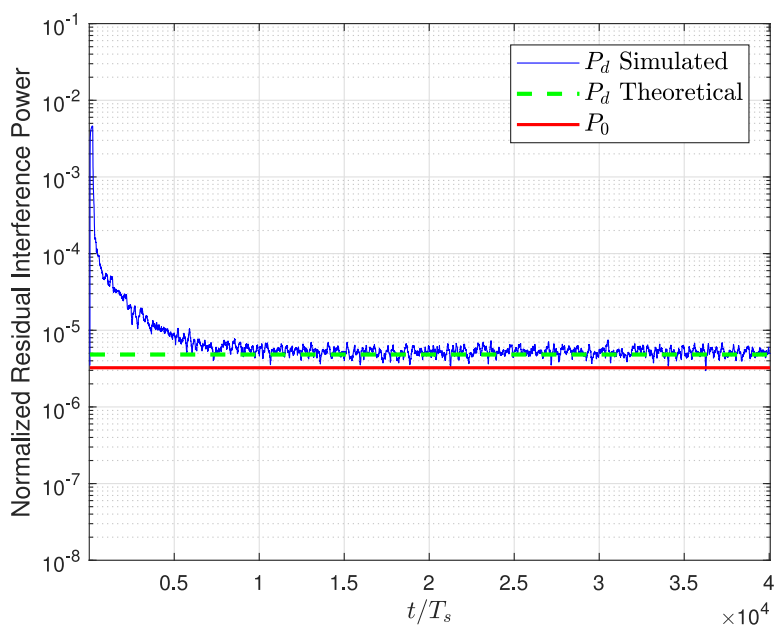

(a)

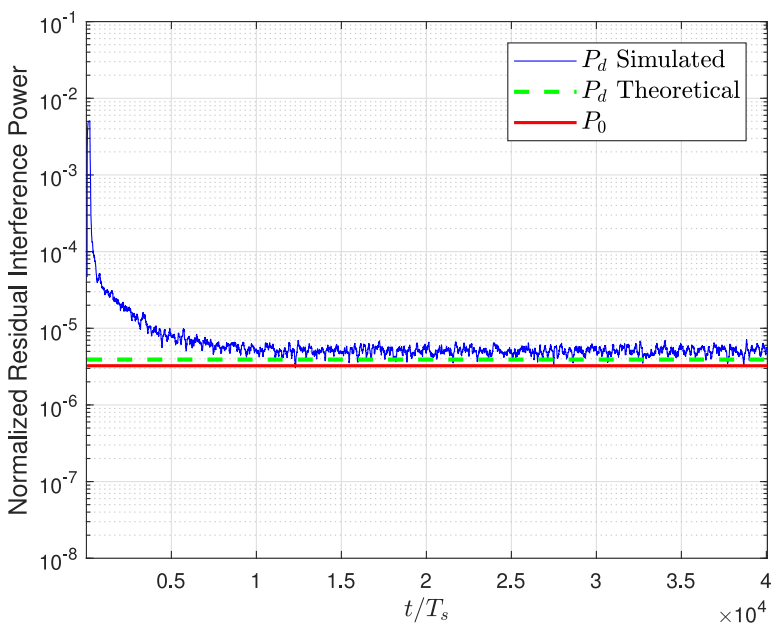

(b)

Fig. 9. Residual SI powers in OFDM system with $\rho_{1}=\rho_{2}=3 \mathrm{~dB}, \theta_{1}=\theta_{2}=5^{\circ}$ and (a) $f_{c}=2432 \mathrm{MHz}$ and (b) $f_{c}=5200 \mathrm{MHz}$.

the steady state analysis. In addition, the gaps between the greendashed lines and the red lines in these figures show the DF of $0.6894 \mathrm{~dB}\left(f_{c}=2432 \mathrm{MHz}\right)$ and $2.426 \mathrm{~dB}\left(f_{c}=5200 \mathrm{MHz}\right)$ in the single-carrier case and $1.7242 \mathrm{~dB}\left(f_{c}=2432 \mathrm{MHz}\right)$ and $0.8067 \mathrm{~dB}\left(f_{c}=5200 \mathrm{MHz}\right)$ in the OFDM case, respectively for the given SI channel.

\section{CONCLUSION}

In this paper, the effects of frequency-independent I/Q imbalances in the ALMS loop have been investigated. It is shown that I/Q imbalance leads to the loop gain variation and reduces the level of SIC provided by the ALMS loop. While the change in the loop gain can be simply compensated by adjusting the gain at other stages inside the loop, the degradation in the level of SIC is proved to be insignificant over numerous observations of the SI channels. The upper bound of this degradation is also derived so that the maximum level of deterioration can be estimated under a given degree of imperfections.

\section{APPENDIX A}

\section{DERIVATION OF RESIDUAL SI POWER}

Substituting (15) into (16), we have

$$
\begin{aligned}
P_{d}(t)= & \frac{1}{K_{1} K_{2}} \bar{E}\left\{\left|\operatorname{Re}\left\{\sum_{l=0}^{L-1} u_{l}^{*}(t) X\left(t-l T_{d}\right) e^{j 2 \pi f_{c} t}\right\}\right|^{2}\right\} \\
= & \frac{1}{4 K_{1} K_{2}} \\
& \cdot \bar{E}\left\{\left[\sum_{l=0}^{L-1} u_{l}^{*}(t) X\left(t-l T_{d}\right) e^{j 2 \pi f_{c} t}+\sum_{l=0}^{L-1} u_{l}(t)\right.\right. \\
& \left.\times X^{*}\left(t-l T_{d}\right) e^{-j 2 \pi f_{c} t}\right]
\end{aligned}
$$




$$
\begin{gathered}
\cdot\left[\sum_{l^{\prime}=0}^{L-1} u_{l^{\prime}}(t) X^{*}\left(t-l^{\prime} T_{d}\right) e^{-j 2 \pi f_{c} t}+\sum_{l^{\prime}=0}^{L-1} u_{l^{\prime}}^{*}(t)\right. \\
\left.\left.\times X\left(t-l^{\prime} T_{d}\right) e^{j 2 \pi f_{c} t}\right]\right\} \\
=\frac{1}{2} \sum_{l=0}^{L-1} \sum_{l^{\prime}=0, l^{\prime} \neq l}^{L-1} \overline{\bar{u}}_{l}^{*}(t) \Theta\left(\left(l-l^{\prime}\right) T_{d}\right) \overline{\bar{u}}_{l^{\prime}}(t) \\
+\frac{1}{2} \Theta(0) \sum_{l=0}^{L-1} \bar{E}\left\{\left|u_{l}(t)\right|^{2}\right\} \\
+\frac{1}{2 K_{1} K_{2}} \bar{E}\left\{\operatorname { R e } \left[\sum_{l=0}^{L-1} u_{l}^{*}(t) X\left(t-l T_{d}\right) \sum_{l^{\prime}=0}^{L-1} u_{l^{\prime}}^{*}(t)\right.\right. \\
\left.\left.\times X\left(t-l^{\prime} T_{d}\right) e^{j 4 \pi f_{c} t}\right]\right\} .
\end{gathered}
$$

Further assuming that the $L$ paths of the SI channel are independent and hence, $u_{l}(t)$ and $u_{l^{\prime}}(t)$ can be considered to be independent for $l \neq l^{\prime}$, (30) becomes

$$
\begin{aligned}
P_{d}(t)= & \frac{1}{2} \sum_{l=0}^{L-1} \sum_{l^{\prime}=0, l^{\prime} \neq l}^{L-1} \overline{\bar{u}}_{l}^{*}(t) \Theta\left(\left(l-l^{\prime}\right) T_{d}\right) \overline{\bar{u}}_{l^{\prime}}(t) \\
& +\frac{1}{2} \Theta(0) \sum_{l=0}^{L-1} \bar{E}\left\{\left|u_{l}(t)\right|^{2}\right\}+\frac{1}{2 K_{1} K_{2}} \\
& \times \bar{E}\left\{\operatorname{Re}\left[\sum_{l=0}^{L-1}\left(u_{l}^{*}(t)\right)^{2} X^{2}\left(t-l T_{d}\right)\right]\right\} .
\end{aligned}
$$

Since $E\left\{X^{2}\right\}=0$ for any zero mean complex random process $X$, we have $\bar{E}\left\{\operatorname{Re}\left[\sum_{l=0}^{L-1}\left(u_{l}^{*}(t)\right)^{2} X^{2}\left(t-l T_{d}\right)\right]\right\}=0$. In addition, from the fact that $E\left\{|X|^{2}\right\} \geq|E\{X\}|^{2}$ for any random process $X$, hence $\bar{E}\left\{\left|u_{l}(t)\right|^{2}\right\} \geq\left|\overline{\bar{u}}_{l}(t)\right|^{2}$. Therefore,

$$
\begin{aligned}
P_{d}(t) \geq & \frac{1}{2} \sum_{l=0}^{L-1} \sum_{l^{\prime}=0, l^{\prime} \neq l}^{L-1} \overline{\bar{u}}_{l}^{*}(t) \Theta\left(\left(l-l^{\prime}\right) T_{d}\right) \overline{\bar{u}}_{l^{\prime}}(t) \\
& +\frac{1}{2} \Theta(0) \sum_{l=0}^{L-1}\left|\overline{\bar{u}}_{l}(t)\right|^{2} \\
= & \frac{1}{2} \sum_{l=0}^{L-1} \sum_{l^{\prime}=0}^{L-1} \overline{\bar{u}}_{l}^{*}(t) \Theta\left(\left(l-l^{\prime}\right) T_{d}\right) \overline{\bar{u}}_{l^{\prime}}(t) .
\end{aligned}
$$

It is also worth noting that the equality happens when $t \rightarrow \infty$ as $u_{l}(t)$ converges to its steady-state value as shown in [20, Eq. (30) and Eq. (35)]. Hence, by letting $t \rightarrow \infty$, we obtain the steady-state $P_{d}$ as in (19).

\section{APPENDIX B}

\section{SOlution OF SteAdy-STATE Weighting ERror Function}

Following the steps presented in [11], [20], [26] to derive the weighting error functions, and substituting the models of $r(t), y(t), x_{1}(t)$, and $x_{2}(t)$ into (6), we have the expression of $w_{l}(t)$ presented in (33) shown at the bottom of the previous page. After filtering out by the LPF, the signal components centered about frequency $2 f_{c}$ will be eliminated. Therefore, (33) becomes

$$
\begin{aligned}
& w_{l}\left(t+t_{0}\right)=\frac{\mu^{\prime} \alpha}{K_{1} K_{2}} \int_{t_{0}}^{t+t_{0}} e^{-\alpha\left(t+t_{0}-\tau\right)}\left\{\left[\sum_{l^{\prime}=0}^{L-1} u_{l^{\prime}}^{*}(\tau) X\left(\tau-l^{\prime} T_{d}\right)\right.\right. \\
& +S(\tau)+N(\tau)] \xi_{1} X^{*}\left(\tau-l T_{d}\right) e^{j 2 \pi f_{c} l T_{d}} \\
& +\left[\sum_{l^{\prime}=0}^{L-1} u_{l^{\prime}}(\tau) X^{*}\left(\tau-l^{\prime} T_{d}\right)\right. \\
& \left.\left.+S^{*}(\tau)+N^{*}(\tau)\right] \eta_{1} X\left(\tau-l T_{d}\right) e^{-j 2 \pi f_{c} l T_{d}}\right\} d \tau
\end{aligned}
$$

$$
\begin{aligned}
& w_{l}\left(t+t_{0}\right)=\frac{2 \mu \alpha}{K_{1} K_{2}} \int_{t_{0}}^{t+t_{0}} e^{-\alpha\left(t+t_{0}-\tau\right)}\{ \frac{1}{2}\left([Z(\tau)+S(\tau)+N(\tau)] e^{j 2 \pi f_{c} t}+\left[Z^{*}(\tau)+S^{*}(\tau)+N^{*}(\tau)\right] e^{-j 2 \pi f_{c} t}\right) \\
&-\frac{1}{2}\left(\sum_{l^{\prime}=0}^{L-1} w_{l^{\prime}}^{*}(t)\left[\eta_{2} X\left(t-l^{\prime} T_{d}\right) e^{j 2 \pi f_{c}\left(t-l^{\prime} T_{d}\right)}+\xi_{2} X^{*}\left(t-l^{\prime} T_{d}\right) e^{-j 2 \pi f_{c}\left(t-l^{\prime} T_{d}\right)}\right]\right. \\
&\left.\left.+\sum_{l^{\prime}=0}^{L-1} w_{l^{\prime}}(t)\left[\eta_{2}^{*} X^{*}\left(t-l^{\prime} T_{d}\right) e^{-j 2 \pi f_{c}\left(t-l^{\prime} T_{d}\right)}+\xi_{2}^{*} X\left(t-l^{\prime} T_{d}\right) e^{j 2 \pi f_{c}\left(t-l^{\prime} T_{d}\right)}\right]\right)\right\} \\
& \times\left[\eta_{1} X\left(\tau-l T_{d}\right) e^{j 2 \pi f_{c}\left(\tau-l T_{d}\right)}+\xi_{1} X^{*}\left(\tau-l T_{d}\right) e^{-j 2 \pi f_{c}\left(\tau-l T_{d}\right)}\right] d \tau \\
&=\frac{\mu \alpha}{K_{1} K_{2}} \int_{t_{0}}^{t+t_{0}} e^{-\alpha\left(t+t_{0}-\tau\right)}\left\{\left[\sum _ { l ^ { \prime } = 0 } ^ { L - 1 } \left(h_{l^{\prime}}-\left[\eta_{2}^{*} w_{l^{\prime}}(\tau)+\xi_{2} w_{l^{\prime}}^{*}(\tau)\right] e^{\left.\left.j 2 \pi f_{c} l^{\prime} T_{d}\right)^{*} X\left(\tau-l^{\prime} T_{d}\right)+S(\tau)+N(\tau)\right] e^{j 2 \pi f_{c} \tau}}\right.\right.\right. \\
&+ {\left.\left[\sum_{l^{\prime}=0}^{L-1}\left(h_{l^{\prime}}-\left[\eta_{2}^{*} w_{l^{\prime}}(\tau)+\xi_{2} w_{l^{\prime}}^{*}(\tau)\right] e^{j 2 \pi f_{c} l^{\prime} T_{d}}\right) X^{*}\left(\tau-l^{\prime} T_{d}\right)+S^{*}(\tau)+N^{*}(\tau)\right] e^{-j 2 \pi f_{c} \tau}\right\} } \\
& \cdot {\left[\eta_{1} X\left(\tau-l T_{d}\right) e^{j 2 \pi f_{c}\left(\tau-l T_{d}\right)}+\xi_{1} X^{*}\left(\tau-l T_{d}\right) e^{-j 2 \pi f_{c}\left(\tau-l T_{d}\right)}\right] d \tau . }
\end{aligned}
$$


Assuming that the signal of interest and the AWGN are independent of the transmitted signal, i.e., $E\left\{S^{*}(t) X(t-\tau)\right\}=0$ and $E\left\{N^{*}(t) X(t-\tau)\right\}=0$ for all $\tau$ and taking ensemble expectation of the two sides of the above equation, we have

$$
\begin{aligned}
& \bar{w}_{l}\left(t+t_{0}\right)=\frac{\mu^{\prime} \alpha}{K_{1} K_{2}} \int_{t_{0}}^{t+t_{0}} e^{-\alpha\left(t+t_{0}-\tau\right)} \\
& \cdot\left\{\left[\sum_{l^{\prime}=0}^{L-1} \bar{u}_{l^{\prime}}^{*}(\tau) E\left\{X\left(\tau-l^{\prime} T_{d}\right) X^{*}\left(\tau-l T_{d}\right)\right\}\right] \xi_{1} e^{j 2 \pi f_{c} l T_{d}}\right. \\
& \left.+\left[\sum_{l^{\prime}=0}^{L-1} \bar{u}_{l^{\prime}}(\tau) E\left\{X^{*}\left(\tau-l^{\prime} T_{d}\right) X\left(\tau-l T_{d}\right)\right\}\right] \eta_{1} e^{-j 2 \pi f_{c} l T_{d}}\right\} d \tau
\end{aligned}
$$

where $\bar{w}_{l}(t)=E\left\{w_{l}(t)\right\}$ and $\bar{u}_{l}(t)=E\left\{u_{l}(t)\right\}$. Defining $\tau^{\prime}=$ $\tau-t_{0}$, we have

$$
\begin{aligned}
& \bar{w}_{l}\left(t+t_{0}\right)=\frac{\mu^{\prime} \alpha}{K_{1} K_{2}} \int_{0}^{t} e^{-\alpha\left(t-\tau^{\prime}\right)}\left\{\left[\sum_{l^{\prime}=0}^{L-1} \bar{u}_{l^{\prime}}^{*}\left(\tau^{\prime}+t_{0}\right)\right.\right. \\
& \left.\cdot E\left\{X\left(\tau^{\prime}+t_{0}-l^{\prime} T_{d}\right) X^{*}\left(\tau^{\prime}+t_{0}-l T_{d}\right)\right\}\right] \xi_{1} e^{j 2 \pi f_{c} l T_{d}} \\
& +\left[\sum_{l^{\prime}=0}^{L-1} \bar{u}_{l^{\prime}}\left(\tau^{\prime}+t_{0}\right) E\left\{X^{*}\left(\tau^{\prime}+t_{0}-l^{\prime} T_{d}\right) X\left(\tau^{\prime}+t_{0}-l T_{d}\right)\right\}\right] \\
& \left.\cdot \eta_{1} e^{-j 2 \pi f_{c} l T_{d}}\right\} d \tau^{\prime} .
\end{aligned}
$$

Taking time average over one symbol period $T$ of $\bar{w}_{l}\left(t+t_{0}\right)$ with respect to the starting time $t_{0}$, we obtain the ensemble and time averaged weighting coefficients as in (37) shown at the bottom the this page.

Since $\alpha$ is very small, $\bar{u}_{l}(t)$ changes slowly and it can be seen as a constant in one symbol period of $T$, i.e., $\bar{u}_{l}\left(t+t_{0}\right) \approx$ $\overline{\bar{u}}_{l}(t)=\frac{1}{T} \int_{0}^{T} \bar{u}_{l}\left(t+t_{0}\right) d t_{0}$ which is the ensemble and time averaged weighting error function $u_{l}(t)$. Thus (37) is expressed as

$$
\begin{aligned}
\overline{\bar{w}}_{l}(t)=\mu^{\prime} \alpha \int_{0}^{t} e^{-\alpha\left(t-\tau^{\prime}\right)} & \left\{\left[\sum_{l^{\prime}=0}^{L-1} \overline{\bar{u}}_{l^{\prime}}^{*}\left(\tau^{\prime}\right) \Theta\left(\left(l^{\prime}-l\right) T_{d}\right)\right\}\right] \\
& \times \xi_{1} e^{j 2 \pi f_{c} l T_{d}} \\
+ & {\left.\left.\left[\sum_{l^{\prime}=0}^{L-1} \overline{\bar{u}}_{l^{\prime}}\left(\tau^{\prime}\right) \Theta\left(\left(l-l^{\prime}\right) T_{d}\right)\right\}\right] \eta_{1} e^{-j 2 \pi f_{c} l T_{d}}\right\} d \tau^{\prime} . }
\end{aligned}
$$

Substituting (38) into the definition of the weighting error function and using the property of the normalized autocorrelation function $\Theta(\tau)=\Theta(-\tau)$, we have

$$
\begin{aligned}
& \overline{\bar{u}}_{l}(t)=h_{l}-\mu^{\prime} \alpha \int_{0}^{t} e^{-\alpha(t-\tau)} \sum_{l^{\prime}=0}^{L-1} \Theta\left(\left(l-l^{\prime}\right) T_{d}\right) \\
& \cdot\left[\left(\eta_{2}^{*} \xi_{1}+\eta_{1}^{*} \xi_{2}\right) \overline{\bar{u}}_{l^{\prime}}^{*}(\tau) e^{j 4 \pi f_{c} l T_{d}}+\left(\eta_{1} \eta_{2}^{*}+\xi_{1}^{*} \xi_{2}\right) \overline{\bar{u}}_{l^{\prime}}(\tau)\right] d \tau
\end{aligned}
$$

or in the matrix form

$$
\begin{aligned}
\overline{\overline{\mathbf{u}}}(t) & =\mathbf{h}-\mu^{\prime} \alpha \int_{0}^{t} e^{-\alpha(t-\tau)} \\
& \cdot\left[\left(\eta_{2}^{*} \xi_{1}+\eta_{1}^{*} \xi_{2}\right) \mathbf{E} \Theta \overline{\overline{\mathbf{u}}}^{*}(\tau)+\left(\eta_{1} \eta_{2}^{*}+\xi_{1}^{*} \xi_{2}\right) \Theta \overline{\overline{\mathbf{u}}}(\tau)\right] d \tau .
\end{aligned}
$$

When $t \rightarrow \infty, \overline{\overline{\mathbf{u}}}(t)$ converges to its steady-state value $\overline{\overline{\mathbf{u}}}$ so that it can be moved outside the integral in (40). Since $\left.\alpha \int_{0}^{t} e^{-\alpha(t-\tau)} d \tau\right|_{t \rightarrow \infty} \rightarrow 1$, (40) becomes

$$
\overline{\overline{\mathbf{u}}}=\mathbf{h}-\mu^{\prime}\left[\left(\eta_{2}^{*} \xi_{1}+\eta_{1}^{*} \xi_{2}\right) \mathbf{E} \Theta \overline{\overline{\mathbf{u}}}^{*}+\left(\eta_{1} \eta_{2}^{*}+\xi_{1}^{*} \xi_{2}\right) \boldsymbol{\Theta} \overline{\overline{\mathbf{u}}}\right],
$$

or

$$
\left[\mathbf{I}_{L}+\mu^{\prime}\left(\eta_{1} \eta_{2}^{*}+\xi_{1}^{*} \xi_{2}\right) \Theta\right] \overline{\overline{\mathbf{u}}}+\mu^{\prime}\left(\eta_{2}^{*} \xi_{1}+\eta_{1}^{*} \xi_{2}\right) \mathbf{E} \Theta \overline{\overline{\mathbf{u}}}^{*}=\mathbf{h} .
$$

Taking complex conjugation on two sides of (42) and noting that $\Theta^{*}=\boldsymbol{\Theta}$ and $\mathbf{E}^{*}=\mathbf{E}^{-1}$, we have

$$
\left[\mathbf{I}_{L}+\mu^{\prime}\left(\eta_{1}^{*} \eta_{2}+\xi_{1} \xi_{2}^{*}\right) \boldsymbol{\Theta}\right] \overline{\overline{\mathbf{u}}}^{*}+\mu^{\prime}\left(\eta_{2} \xi_{1}^{*}+\eta_{1} \xi_{2}^{*}\right) \mathbf{E}^{-1} \Theta \overline{\overline{\mathbf{u}}}=\mathbf{h}^{*}
$$

From (42) and (43), we have a set of equations

$$
\left\{\begin{array}{l}
\left(\mathbf{I}_{L}+k_{1} \boldsymbol{\Theta}\right) \overline{\overline{\mathbf{u}}}+k_{2} \mathbf{E} \Theta \overline{\overline{\mathbf{u}}}^{*}=\mathbf{h} \\
k_{2}^{*} \mathbf{E}^{-1} \boldsymbol{\Theta} \overline{\overline{\mathbf{u}}}+\left(\mathbf{I}_{L}+k_{1}^{*} \boldsymbol{\Theta}\right) \overline{\overline{\mathbf{u}}}^{*}=\mathbf{h}^{*}
\end{array}\right.
$$

where $k_{1}=\mu^{\prime}\left(\eta_{1} \eta_{2}^{*}+\xi_{1}^{*} \xi_{2}\right)$ and $k_{2}=\mu^{\prime}\left(\eta_{2}^{*} \xi_{1}+\eta_{1}^{*} \xi_{2}\right)$. From the second equation in (44), we can expressed $\overline{\overline{\mathbf{u}}}^{*}$ as

$$
\overline{\overline{\mathbf{u}}}^{*}=\left(\mathbf{I}_{L}+k_{1}^{*} \boldsymbol{\Theta}\right)^{-1}\left[\mathbf{h}^{*}-k_{2}^{*} \mathbf{E}^{-1} \boldsymbol{\Theta} \overline{\overline{\mathbf{u}}}\right] .
$$

Substituting (45) into the first equation in (44), we obtain the solution for the weighting error functions as in (20).

$$
\begin{aligned}
\overline{\bar{w}}_{l}(t)=\frac{1}{T} \int_{0}^{T} \bar{u}\left(t+t_{0}\right) d t_{0}= & \frac{\mu^{\prime} \alpha}{K_{1} K_{2} T} \int_{0}^{T} \int_{0}^{t} e^{-\alpha\left(t-\tau^{\prime}\right)}\left\{\left[\sum_{l^{\prime}=0}^{L-1} \bar{u}_{l^{\prime}}^{*}\left(\tau^{\prime}+t_{0}\right) E\left\{X\left(\tau^{\prime}+t_{0}-l^{\prime} T_{d}\right) X^{*}\left(\tau^{\prime}+t_{0}-l T_{d}\right)\right\}\right] \xi_{1} e^{j 2 \pi f_{c} l T_{d}}\right. \\
& \left.+\left[\sum_{l^{\prime}=0}^{L-1} \bar{u}_{l^{\prime}}\left(\tau^{\prime}+t_{0}\right) E\left\{X^{*}\left(\tau^{\prime}+t_{0}-l^{\prime} T_{d}\right) X\left(\tau^{\prime}+t_{0}-l T_{d}\right)\right\}\right] \eta_{1} e^{-j 2 \pi f_{c} l T_{d}}\right\} d \tau^{\prime} d t_{0}
\end{aligned}
$$




\section{REFERENCES}

[1] T. Le-Ngoc and A. Masmoudi, Full-Duplex Wireless Communications Systems. Berlin, Germany: Springer, 2017.

[2] L. Song, R. Wichman, Y. Li, and Z. Han, Full-Duplex Communications and Networks. Cambridge, U.K: Cambridge Univ. Press, 2017.

[3] Y. Nan, X. Huang, and Y. J. Guo, "Generalized continuous wave synthetic aperture radar for high resolution and wide swath remote sensing," IEEE Trans. Geosci. Remote Sens., vol. 56, no. 12, pp. 7217-7229, Dec. 2018.

[4] C. D. Nwankwo, L. Zhang, A. Quddus, M. A. Imran, and R. Tafazolli, "A survey of self-interference management techniques for single frequency full duplex systems," IEEE Access, vol. 6, pp. 30 242-30 268, 2017.

[5] C. R. Anderson et al., "Antenna isolation, wideband multipath propagation measurements, and interference mitigation for on-frequency repeaters," in Proc. IEEE SoutheastCon, Greensboro, North Carolina, USA, Mar. 2004, pp. 110-114.

[6] E. Everett, A. Sahai, and A. Sabharwal, "Passive self-interference suppression for full-duplex infrastructure nodes," IEEE Trans. Wireless Commun., vol. 13, no. 2, pp. 680-694, Feb. 2014.

[7] M. Duarte, C. Dick, and A. Sabharwal, "Experiment-driven characterization of full-duplex wireless systems," IEEE Trans. Wireless Commun., vol. 11, no. 12, pp. 4296-4307, Dec. 2012.

[8] J. Zhou and H. Krishnaswamy, "Recent developments in fully-integrated RF self-interference cancellation for frequency-division and full-duplex radios," in Proc. 81st IEEE Veh. Technol. Conf., Glasgow, U.K., 2015, pp. $1-5$.

[9] D. Korpi, L. Anttila, V. Syrjälä, and M. Valkama, "Widely linear digital self-interference cancellation in direct-conversion full-duplex transceiver," IEEE J. Sel. Areas Commun., vol. 32, no. 9, pp. 1674-1687, Sep. 2014.

[10] Z. Zhang, K. Long, A. V. Vasilakos, and L. Hanzo, "Full-duplex wireless communications: Challenges, solutions, and future research directions," Proc. IEEE, vol. 104, no. 7, pp. 1369-1409, Jul. 2016.

[11] A. T. Le, L. C. Tran, X. Huang, Y. J. Guo, and J. C. Vardaxoglou, "Frequency domain characterization and performance bounds of ALMS loop for RF self-interference cancellation," IEEE Trans. Commun., vol. 67, no. 1, pp. 682-692, Jan. 2019.

[12] D. Liu, Y. Shen, S. Shao, Y. Tang, and Y. Gong, "On the analog selfinterference cancellation for full-duplex communications with imperfect channel state information," IEEE Access, vol. 5, pp. 9277-9290, 2017

[13] M. Duarte and A. Sabharwal, "Full-duplex wireless communications using off-the-shelf radios: Feasibility and first results," in Proc. 44th Conf. Rec. Asilomar Conf. Signals, Syst. Comput., Pacific Grove, CA, USA, 2010, pp. $1558-1562$.

[14] A. Kiayani et al., "Adaptive nonlinear RF cancellation for improved isolation in simultaneous transmit-receive systems," IEEE Trans. Microw. Theory Techn., vol. 66, no. 5, pp. 2299-2312, Jan. 2018.

[15] D. Korpi et al., "Full-duplex mobile device: Pushing the limits," IEEE Commun. Mag., vol. 54, no. 9, pp. 80-87, Sep. 2016.

[16] T. Huusari, Y. S. Choi, P. Liikkanen, D. Korpi, S. Talwar, and M. Valkama, "Wideband self-adaptive RF cancellation circuit for full-duplex radio: Operating principle and measurements," in Proc. 81st IEEE Veh. Technol. Conf., Glasgow, Scotland, 2015, pp. 11-14

[17] Y. S. Choi and H. Shirani-Mehr, "Simultaneous transmission and reception: Algorithm, design and system level performance," IEEE Trans. Wireless Commun., vol. 12, no. 12, pp. 5992-6010, Oct. 2013.

[18] K. E. Kolodziej, J. G. McMichael, and B. T. Perry, "Multitap RF canceller for in-band full-duplex wireless communications," IEEE Trans. Wireless Commun., vol. 15, no. 6, pp. 4321-4334, Jun. 2016.

[19] M. Sakai, H. Lin, and K. Yamashita, "Self-interference cancellation in full-duplex wireless with IQ imbalance," Phys. Commun., vol. 18, no. P1, pp. 2-14, Mar. 2016.

[20] X. Huang and Y. J. Guo, "Radio frequency self-interference cancellation with analog least mean-square loop," IEEE Trans. Microw. Theory Tech., vol. 65, no. 9, pp. 3336-3350, Sep. 2017.

[21] A. T. Le, L. C. Tran, and X. Huang, "Cyclostationary analysis of analog least mean square loop for self-interference cancellation in in-band fullduplex systems," IEEE Commun. Lett., vol. 21, no. 12, pp. 2738-2741, Sep. 2017.

[22] A. T. Le, L. C. Tran, and X. Huang, "On performance of analog least mean square loop for self-interference cancellation in in-band full-duplex OFDM systems," in Proc. 85th IEEE Veh. Technol. Conf., Sydney, Australia, 2017, pp. 1-5.
[23] Y. Pan, C. Zhou, G. Cui, W. Wang, and X. Li, "Self-interference cancellation with RF impairments suppression for full-duplex systems," in Proc. 82nd IEEE Veh. Technol. Conf., Boston, USA, Sep. 2015, pp. $1-5$.

[24] F. Shu, J. Wang, J. Li, R. Chen, and W. Chen, "Pilot optimization, channel estimation, and optimal detection for full-duplex OFDM systems with IQ imbalances," IEEE Trans. Veh. Technol., vol. 66, no. 8, pp. 6993-7009, Aug. 2017.

[25] H. Yu et al., "Compressed sensing-based time-domain channel estimator for full-duplex OFDM systems with IQ-imbalances," Sci. China Inf. Sci., vol. 60, no. 8, Mar. 2017, Art. no. 082303. [Online]. Available: https://doi.org/10.1007/s11432-016-0386-x

[26] A. T. Le, Y. Nan, L. C. Tran, X. Huang, Y. J. Guo, and Y. Vardaxoglou, "Analog least mean square loop for self-interference cancellation in generalized continuous wave SAR," in Proc. 88th IEEE Veh. Technol. Conf., Chicago, USA, 2018, pp. 1-5.

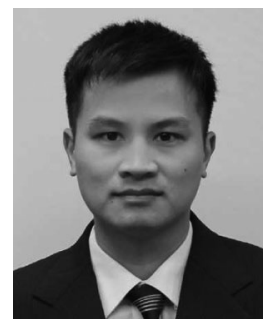

Anh Tuyen Le received the B. Eng. and M. Eng. degrees in telecommunication engineering from Le Quy Don Technical University, Hanoi, Vietnam and University of Wollongong, Wollongong, NSW, Australia, in 2008 and 2014, respectively. He is currently working toward the $\mathrm{Ph} . \mathrm{D}$. degree in engineering with the Global Big Data Technologies Center, University of Technology Sydney, Sydney, NSW, Australia. His research interests include signal processing, circuits and systems for RF and millimetre applications.

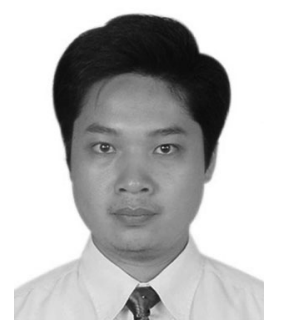

Le Chung Tran (M'09-SM'18) received the B.E. (1st Hons., HD), M.E. (HD), and Ph.D. degrees from the University of Transport and Communication (UTC), Hanoi, Vietnam, University of Science and Technology, Hanoi, Vietnam, and University of Wollongong (UOW), Wollongong, NSW, Australia, in 1997,2000 , and 2006, respectively, all in telecommunications engineering. He was a Lecturer with UTC from 1997 to 2012. From 2005 to 2006, he was an Associate Research Fellow with the Wireless Technologies Laboratory, UOW. During 2006-2008, he was a Postdoctoral Research Fellow with the University of Luebeck, Germany, under the Alexander von Humboldt $(\mathrm{AvH})$ fellowship. He has been with the UOW since 2009 to date, where he is currently a Senior Lecturer. He is a Co-Author of 83 publications, including one book, which is hold in 337 worldwide libraries. His research interests include 5G, MIMO, space-time-frequency processing, WBANs, IoT, biomedical engineering, ultra-wideband, millimetre wave, cooperative and cognitive communications, software defined radio, network coding, and digital signal processing for communications. He was the recipient of the World University Services (WUS) awards (twice), Vietnamese Government's Doctoral Scholarship, International Postgraduate Research Scholarship (IPRS), and the prestigious Humboldt Fellowships (twice). He has served as an Advisory Board Member for Cambridge Scholars Publishing; an Editorial Board Member of the Electrical Engineering: An International Journal (EEIJ); an Organizing Committee Member (Track Chair, Session Chair, Publicity Co-Chair) and a Technical Program Committee (TPC) Member for more than 30 IEEE conferences. 


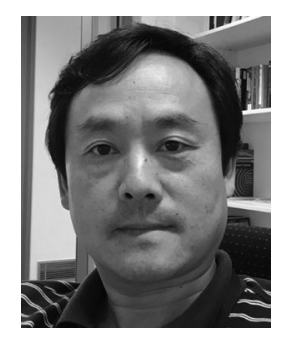

Xiaojing Huang (M'99-SM'11) received the B.Eng., M.Eng., and Ph.D. degrees in electronic engineering from Shanghai Jiao Tong University, Shanghai, China, in 1983, 1986, and 1989, respectively. He was a Principal Research Engineer with the Motorola Australian Research Center, Botany, NSW, Australia, from 1998 to 2003, and an Associate Professor with the University of Wollongong, Wollongong, NSW, Australia, from 2004 to 2008. He had been a Principal Research Scientist with the Commonwealth Scientific and Industrial Research Organisation (CSIRO), Sydney, NSW, Australia, and the Project Leader of the CSIRO Microwave and mmWave Backhaul projects since 2009. He is currently a Professor of Information and Communications Technology with the School of Electrical and Data Engineering and the Program Leader for Mobile Sensing and Communications with the Global Big Data Technologies Center, University of Technology Sydney, Sydney, NSW, Australia. With more than 30 years of combined industrial, academic, and scientific research experience, he has authored more than 300 book chapters, refereed journal and conference papers, major commercial research reports, and filed 31 patents. He was the recipient of the CSIRO Chairman's Medal and the Australian Engineering Innovation Award in 2012 for exceptional research achievements in multigigabit wireless communications.

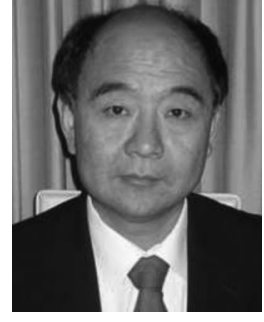

Yingiie Jay Guo (F'14) received the bachelor's degree and a master's degree from Xidian University, Xi' an, China, in 1982 and 1984, respectively, and the $\mathrm{Ph} . \mathrm{D}$. degree from Xian Jiaotong University, Xi'an, China, in 1987.

$\mathrm{He}$ is a Distinguished Professor and the Founding Director of Global Big Data Technologies Centre with the University of Technology Sydney, Sydney, NSW, Australia. Prior to this appointment in 2014, he was the Director with CSIRO for more than nine years. Before joining CSIRO, he held various senior technology leadership positions in Fujitsu, Siemens, and NEC in the U.K. His research interest includes antennas, $\mathrm{mm}$-wave and $\mathrm{THz}$ communications and sensing systems as well as big data technologies. He has authored more 450 research papers including 210 journal papers and holds 26 patents in antennas and wireless systems. Prof. Guo has chaired numerous international conferences. He is the Chair Elect of International Steering Committee, International Symposium on Antennas and Propagation. He was the International Advisory Committee Chair of the IEEE VTC2017, General Chair of ISAP2015, iWAT2014, and WPMC'2014, and TPC Chair of 2010 IEEE WCNC, and 2012 and 2007 IEEE ISCIT. He was the Guest Editor of special issues on "Antennas for Satellite Communications" and "Antennas and Propagation Aspects of 60-90GHz Wireless Communications," both in the IEEE TRANSACTIONS ON ANTENNAS AND PROPAGation, Special Issue on "Communications Challenges and Dynamics for Unmanned Autonomous Vehicles," the IEEE JOURNAL ON SeleCted AREAS IN COMMUNiCATIONS, and Special Issue on "5G for Mission Critical Machine Communications," the IEEE NETwORK MAGAZINE. He is a Fellow of the Australian Academy of Engineering and Technology and IET, and was a member of the College of Experts of Australian Research Council (ARC, 2016-2018). He has won a number of most prestigious Australian engineering and CSIRO awards, and was named one of the most influential engineers in Australia in 2014 and 2015, respectively. 